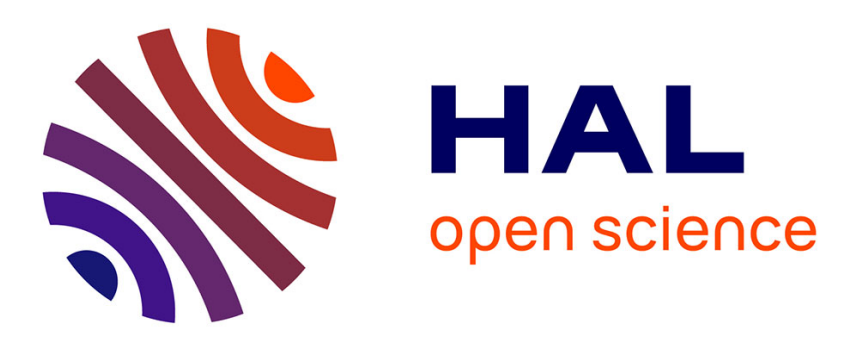

\title{
Large eddy simulation and measurements of turbulent enclosed rotor-stator flows
}

Eric Séverac, Sébastien Poncet, Eric Serre, Marie-Pierre Chauve

\section{To cite this version:}

Eric Séverac, Sébastien Poncet, Eric Serre, Marie-Pierre Chauve. Large eddy simulation and measurements of turbulent enclosed rotor-stator flows. Physics of Fluids, 2007, 19 (8), pp.085113. 10.1063/1.2759530 . hal-00170072

\section{HAL Id: hal-00170072 \\ https://hal.science/hal-00170072}

Submitted on 6 Sep 2007

HAL is a multi-disciplinary open access archive for the deposit and dissemination of scientific research documents, whether they are published or not. The documents may come from teaching and research institutions in France or abroad, or from public or private research centers.
L'archive ouverte pluridisciplinaire HAL, est destinée au dépôt et à la diffusion de documents scientifiques de niveau recherche, publiés ou non, émanant des établissements d'enseignement et de recherche français ou étrangers, des laboratoires publics ou privés. 


\title{
Large Eddy Simulation and Measurements of Turbulent Enclosed Rotor-Stator Flows
}

\author{
Éric Séverac, Sébastien Poncet, ${ }^{*}$ and Éric Serre \\ Laboratoire de Modélisation et Simulation Numérique en Mécanique - Génie des Procédés \\ UMR 6181 CNRS - Universités d'Aix-Marseille I, II \& III \\ IMT La Jetée, Technopôle Château-Gombert, \\ 38 rue F. Joliot-Curie. 13451 Marseille cédex 20 - FRANCE - Fax. 33 (4) 91118502 \\ Marie-Pierre Chauve \\ Institut de Recherche sur les Phénomènes Hors Équilibre \\ UMR 6594 CNRS - Universités d'Aix-Marseille I \& II \\ Technopôle Château-Gombert, 49 rue F. Joliot-Curie, \\ BP 146. 13384 Marseille cédex 13 - FRANCE - Fax. 33 (4) 96139709
}

(Dated: June 16, 2007)

Turbulent flows are studied in an actual enclosed rotor-stator configuration with a rotating hub and a stationary shroud. Besides its fundamental importance - the disk boundary layer is one of the simplest platforms for investigating the underlying structure of three-dimensional boundary layers - this cavity models more complex configurations relevant to rotating machinery. Large Eddy Simulation (LES) is performed using a Spectral Vanishing Viscosity (SVV) technique which is shown leading to stable discretizations without sacrificing the formal accuracy of the spectral approximation. Numerical results and velocity measurements have been favorably compared for a large range of rotational Reynolds numbers $10^{5} \leq R e=\Omega b^{2} / \nu \leq 10^{6}$ in an annular cavity of curvature parameter $R_{m}=(b+a) /(b-a)=1.8$ and of aspect ratio $G=(b-a) / h=5$, where $a$ and $b$ are respectively the inner and outer radii of the rotating disk and $h$ is the interdisk spacing.

In the detailed picture of the flow structure that emerges, the turbulence is mainly confined in the boundary layers including in the Stewartson layer along the external cylinder. For Reynolds numbers $R e \geq 10^{5}$, the stator boundary layer is turbulent over most of the cavity. On the other hand, the rotor layer becomes progres- 
sively turbulent from the outer radial locations although the rotating hub is shown to destabilize the inner part of the boundary layers. The isosurface maps of the Q-criterion reveal that the three-dimensional spiral arms observed in the unstable laminar regime evolve to more axisymmetric structures when turbulence occurs. At $R e=10^{6}$, the flow is fully turbulent and the anisotropy invariant map highlights turbulence structuring, which can be either a "cigar-shaped" structuring aligned on the tangential direction or a "pancake-shaped" structuring depending on the axial location. The reduction of the structural parameter $a_{1}$ (the ratio of the magnitude of the shear stress vector to twice the turbulence kinetic energy) under the typical limit 0.15 , as well as the misalignment between the shear stress vector and the mean velocity gradient vector, highlight the three-dimensional nature of both rotor and stator boundary layers with a degree of three-dimensionality much higher than in the idealized system studied by Lygren and Andersson [1, 21, 22].

Keywords: rotor-stator flow, large eddy simulation, spectral vanishing viscosity technique, LDV.

*Electronic address: poncet@13m.univ-mrs.fr, Tel.33(4)91.11.85.23 


\section{INTRODUCTION}

Rotating disk flows have been the subject of a constant interest because of their relevance to applications in rotating machinery systems as computer storage, axial thrust bearings and turbine disk cooling. The rotor-stator problem has also proved to be a fruitful means of studying turbulence properties with wall confinement and rotation as this specific configuration is a relatively simple case where rotation brings significant modifications to the turbulent field. Finally, rotating disk flows are also among the simplest flows where the boundary layers are three-dimensional from their inception and they are therefore well suited for studying the effects of mean-flow three-dimensionality on the turbulence and its structure $[14,19,21]$. Until now, numerical studies have been dedicated to simpler flows: single disk flows [44], axisymmetric flows using statistical approaches (Reynolds Averaged Navier-Stokes) [27], idealized rotor-stator cavities [21], or enclosed rotor-stator cavities but at much lower rotation rates using Direct Numerical Simulation (DNS) [28, 35]. The aim of this study is to provide a detailed and accurate picture of the turbulent flow for understanding the physics as well as for the assessment of turbulence models for rotating flow systems.

Rotor-stator flow structure is largely dependent on the combination of the rotation speed $\Omega$ and the interdisk spacing $h$. Daily and Nece [7] carried out an exhaustive theoretical and experimental study of sealed rotor-stator flows and pointed out the existence of four flow regimes. These correspond respectively to two laminar regimes (I and II) and two turbulent regimes (III and IV), each characterized by either merged (I and III) or separated (II and IV) boundary layers. In the latter case, the two boundary layers are separated by a central rotating core. These authors provided also an estimated value for the local Reynolds number at which turbulence originates with separated boundary layers, $R e_{r}=\Omega r^{2} / \nu=1.5 \times 10^{5}(r$ is the radial location) for aspect ratios $G \leq 25$. However, experiments have revealed that transition to turbulence can appear at a lower value of $R e_{r}$ within the Bödewadt layer on the stator, even though the flow remains laminar in the Ekman layer along the rotor.

Major experiments concerning this flow regime have been performed by Itoh et al. $[11,12]$ in a closed cavity. They measured the mean flow and all the Reynolds stress components, and brought out the existence of a relaminarized region towards the axis even at high rotation rates. From detailed measurements, Itoh et al. [12] reported a turbulent regime occurring earlier along the stator side at $R e_{r} \simeq 8 . \times 10^{3}$, while along the rotor side, turbu- 
lent flow develops later for $3.6 \times 10^{5}<R e_{r}<6.4 \times 10^{5}$ for $G=12.5$. This is in agreement with the experiment of Cheah et al. [4] performed for rotational Reynolds numbers ranging $3 \times 10^{5}<R e_{r}<1.6 \times 10^{6}$ inside a rotor-stator system of aspect ratio $G=7.87$. The rotor side becomes turbulent for $R e_{r}=4 . \times 10^{5}$ while the stator is shown to be turbulent at all Reynolds numbers considered. Cheah et al. [4] found differences in turbulence characteristics between the rotor and the stator and that the turbulent flow field is also affected by the radial location. These authors concluded to an influence of the radial convective transport of turbulence on the flow field. Czarny et al. [6] displayed by flow visualizations the appearance of organized precessing vortex structures in a rotor-stator cavity of aspect ratio $G=7.94$. These three-dimensional patterns subsist at high Reynolds numbers $R e \rightarrow 1.78 \times 10^{6}$ and might explain the failure to predict heat and mass transfers accurately when only axisymmetric and steady approaches are used. More recently, Poncet et al. [27] compared extensive pressure and velocity measurements with numerical predictions based on an improved version of the Reynolds Stress Modeling (RSM) of Elena and Schiestel [8] for an enclosed cavity with or without superimposed throughflow. In the case of an outward throughflow, they characterized the transition between the Batchelor and Stewartson flow structures in function of a modified Rossby number.

These flows are very challenging for numerical modeling particularly in turbulent regimes relevant to industrial conditions. A characteristic feature of such flows is indeed the coexistence of adjacent coupled flow regions involving laminar, transitional and turbulent regions completely different in terms of the flow properties. Moreover, the turbulence is strongly inhomogeneous and anisotropic because of finite cavity effects, flow curvature and rotational effects. At present, computer performances only permit DNS of transitionally turbulent cavity flows $\left(R e \approx 10^{5}\right)[27,35]$. In a simpler flow model, where the flow is restricted to an angular section of the cavity and assumed homogeneous also in the radial direction (assuming slow variation of the turbulence statistics in the radial direction far from the transition), Lygren and Andersson [21] performed DNS at higher Reynolds number $\left(\operatorname{Re}=4 \times 10^{5}\right)$ using a second-order finite-difference scheme. They provided a detailed set of data to analyze the coherent structures near the two disks. Attempts to compute turbulent rotor-stator flows using statistical approaches (Reynolds Averaged Navier-Stokes) had only partial success. Indeed, the turbulence model must be able to solve the low-Reynolds number region not only near the disks but also in the core of the flow. Moreover, the model has to predict pre- 
cisely the location of the transition from the laminar to the turbulent regime, even though it is bounded by instabilities, and so cannot be completely represented by a steady flow model. The second order closures could be a more appropriate level of closure to predict such complex flows $[17,27]$ but even if they provide a correct distribution of laminar and turbulent regions, the Reynolds stress behaviour is not fully satisfactory, particularly near the rotating disk and in the core region.

Consequently, Large Eddy Simulation (LES) constitutes a valuable way to compute such flows. Wu and Squires [44] have been the first to develop LES to predict the statistically three-dimensional turbulent boundary layer (3DTBL) over a single rotating disk. They compared three dynamical subgrid models to the experimental data of Littell and Eaton [19] for $R e=6.5 \times 10^{5}$. Their results have offered new evidence to support the observations of Littell and Eaton [19] that streamwise vortices with the same sign as the mean streamwise vorticity are mostly responsible for strong sweep events, while streamwise vortices having opposite sign to the mean streamwise vorticity promote strong ejections. Lygren and Andersson [22] compared the results obtained from three LES models with their DNS calculation and suggested that improved subgrid models have to be implemented to get closer agreement. In their latter work, Andersson and Lygren [1] performed "wide-gap" and "narrow-gap" simulations to investigate the degree of three-dimensionality in both Ekman and Bödewadt layers for $R e \leq 1.6 \times 10^{6}$. Their results support the same conclusions as Littell and Eaton [19] that the mean flow three-dimensionality affects the near wall vortices and their ability to generate shear-stresses.

We report here a coupled numerical and experimental investigation of the turbulent flow within an enclosed rotor-stator cavity. A spectral vanishing viscosity has been used to perform Large Eddy Simulation by modifying Navier-Stokes equations in order to obtain a new system of equations which is more amenable to approximate while retaining all the most energetic features of the unperturbed problem. The main motivation of our study is to analyze turbulence properties when complex effects from rotation and confinement may influence the near-wall structures caused by the mean three-dimensionality. As confinement effects are fully taken into account, this work represents a further step compared to existing LES works dealing with turbulent rotating flows.

First experimental set-up and numerical modeling are described in Sections II and III. Comparisons between the LES calculations and the LDV measurements are made at mid- 
radius in Section IV for the mean and turbulent fields at different Reynolds numbers ranging $10^{5} \leq R e=\Omega b^{2} / \nu \leq 10^{6}$. Section $\mathrm{V}$ is devoted to the analyze of finite cavity effects at different radial locations and at $R e=10^{6}$. A detailed investigation of the boundary layers along the disks and the external cylinder is then proposed in section VI. Flow structures are identified in Section VII. Finally some conclusions and closing remarks are provided in section VIII.

\section{THE EXPERIMENTAL SET-UP}

The cavity sketched in Figure 1 is composed of a smooth stationary disk (the stator) and a smooth rotating disk (the rotor) delimited by an inner rotating cylinder (the hub) and an outer stationary casing (the shroud). The rotor and the central hub attached to it rotate at the same uniform angular velocity $\Omega$. The mean flow is governed by three main control parameters: the aspect ratio of the cavity $G$, the curvature parameter $R_{m}$ and the rotational Reynolds number $R e$ based on the outer radius $b$ of the rotating disk defined as follows:

$$
G=\frac{b-a}{h}=5 \quad R_{m}=\frac{b+a}{b-a}=1.8 \quad 10^{5} \leq R e=\frac{\Omega b^{2}}{\nu} \leq 10^{6}
$$

where $\nu$ is the kinematic viscosity of water, $a=40 \mathrm{~mm}$ and $b=140 \mathrm{~mm}$ the inner and outer radii of the rotating disk, $h=20 \mathrm{~mm}$ the interdisk spacing and $\Omega$ the rotation rate of the rotating disk. The values of the geometrical parameters have been chosen in order to be relevant with industrial devices such as real stage of turbopump, and to satisfy technical constraints of the experimental device as well as computational effort to reach statistically converged stages. A variable speed numerical controller drives the angular velocity $\Omega$ with an accuracy better than $1 \%$. In the experimental set-up, two small clearances exist: the first one $\delta_{1}=0.85 \mathrm{~mm}$ between the rotor and the shroud $\left(\delta_{1} / b=0.0061\right)$ and the second one $\delta_{2}=0.1 \mathrm{~mm}$ between the hub and the stator $\left(\delta_{2} / h=0.005\right)$ because of mechanical constraints. During one experiment, the temperature is almost constant: $20 \pm 0.5^{\circ} \mathrm{C}$.

The measurements are performed using a two component laser Doppler velocimeter (LDV). The LDV technique is used to measure from above the stator the mean radial $V_{r}^{*}=V_{r} /(\Omega r)$ and tangential $V_{\theta}^{*}=V_{\theta} /(\Omega r)$ velocity components and the associated Reynolds stress tensor components $R_{r r}^{*}=\overline{v_{r}^{\prime 2}} /(\Omega r)^{2}, R_{r \theta}^{*}=\overline{v_{r}^{\prime} v_{\theta}^{\prime}} /(\Omega r)^{2}, R_{\theta \theta}^{*}=\overline{v_{\theta}^{\prime 2}} /(\Omega r)^{2}$ 
in a vertical plane $(r, z)$. This method is based on the accurate measurement (error margin of $\pm 5 \%$ on the second order momentums) of the Doppler shift of laser light scattered by small particles (Optimage PIV Seeding Powder, $30 \mu \mathrm{m}$ ) carried along with the fluid. Its main qualities are its non intrusive nature and its robustness. The measurement is found to be more difficult close to the rotating disk, because of strong reflections of the laser beams and seeding problems. Note that the size of the probe volume in the axial direction $(0.8 \mathrm{~mm})$ is not small compared to the interdisk spacing and to the boundary layer thicknesses. It has been experimentally verified [25] that about 5000 validated data are necessary to obtain the statistical convergence of the velocity fluctuations.

\section{THE NUMERICAL MODELING}

A spectral vanishing viscosity (SVV) method for LES has been developed for simulating rotor-stator flows. It has the property to preserve the spectral accuracy of the approximation developed in DNS [37] and keeps the fast time integration of the DNS because it is condensed in pre-processing jobs. The reader is referred to the original paper of Karamanos and Kardianakis [15] and in the collocation framework for cylindrical equations to the paper of Séverac and Serre [38].

\section{A. Governing equations}

The motion is governed by the incompressible three-dimensional Navier-Stokes equations written in the velocity-pressure formulation, together with the continuity equation and appropriate boundary conditions. A cylindrical polar coordinate system $(r, \theta, z)$ is used. The velocity components are denoted $V_{r}, V_{\theta}$ and $V_{z}$ and $p$ is the pressure. The velocity, space and time scalings correspond to $\Omega b, h$ and $\Omega^{-1}$ respectively. In the meridional plane, the space variables $(r, z) \in[a, b] \times[0, h]$ have been normalized into the square $[-1,1] \times[-1,1]$, a prerequisite for the use of Chebyshev polynomials. 


\section{B. Numerical method}

The pseudospectral numerical method is based on a collocation-Chebyshev method in the $r$ and $z$ non-homogeneous directions and a Galerkin-Fourier method in the azimuthal periodic direction $\theta$. Thus, each dependent variable $f=\left(V_{r}, V_{\theta}, V_{z}, p\right)$ is expanded into a truncated trigonometric series:

$$
f_{N M K}(r, \theta, z, t)=\sum_{n=0}^{N-1} \sum_{m=0}^{M-1} \sum_{k=-K / 2}^{K / 2-1} \hat{f}_{n m k}(t) T_{n}(r) T_{m}(z) e^{i k \theta}
$$

where $T_{n}$ and $T_{m}$ are Chebyshev polynomials of degrees $n$ and $m$ respectively. $N, M$ define the number of collocation points in the radial and axial directions, respectively and $K$ is the cutoff in the tangential direction. To ensure high accuracy of the solution within the very thin wall layers, this approximation is applied at the Gauss-Lobatto collocation points, where the differential equations are assumed to be satisfied exactly, defined as $r_{i}=\cos \left(\frac{i \pi}{N}\right)$ for $i \in[0, N]$ and $z_{j}=\cos \left(\frac{j \pi}{M}\right)$ for $j \in[0, M]$ in the radial and axial directions. In the azimuthal direction, an uniform distribution is considered: $\theta_{k}=2 k \pi / K$ for $k \in[0, K[$.

The time scheme is semi-implicit and second order accurate. It is a combination of an explicit treatment of the convective terms (Adams-Bashforth scheme) and of an implicit treatment for the diffusive terms (second order backward Euler scheme). The solution method is based on an efficient projection scheme to solve the coupling between velocity and pressure (see details in [29]). This algorithm ensures a divergence-free velocity field at each time step, maintains the order of accuracy of the time scheme for each dependent variable and does not require the use of staggered grids. Finally, for each Fourier mode, a full diagonalization technique is used and yields simple matrix products for the solution of successive 2D uncoupled Helmholtz and Poisson equations at each time step.

The Spectral Vanishing Viscosity (SVV), first introduced by Tadmor [40] for stabilizing the solution of the inviscid Bürgers equation, is incorporated into the cylindrical NavierStokes equations. A viscosity kernel operator, only active for high wave numbers of the numerical approximation, is incorporated in the Helmholtz equations of velocity prediction [38]. Then, the diffusion and SVV terms are combined in order to obtain a new diffusion operator that can be easily written in $1 \mathrm{D}$ :

$$
\nu \Delta_{S V V} v_{N}=\nu \Delta v_{N}+\varepsilon_{N} \partial x\left(Q_{N} \cdot \frac{\partial v_{N}}{\partial x}\right)
$$


where $v_{N}$ is the velocity vector approximation and $Q_{N}$ is the kernel defined in the spectral space as:

$$
\hat{Q_{N}}\left(w_{n}\right)= \begin{cases}0 & 0 \leq w_{n} \leq w_{T} \\ \epsilon_{N} \cdot e^{-\left(\frac{w_{N}-w_{n}}{w_{T}-w_{n}}\right)^{2}} & w_{T}<w_{n} \leq w_{N}\end{cases}
$$

where $\epsilon_{N}$ is the maximum of viscosity, $w_{T}$ is the mode after which the spectral viscosity is applied and $w_{N}$ the highest mode calculated. Thus, the viscosity kernel is zero on the lower frequencies. There is no direct way to extend the one dimensional definition of the SVV operator to the three-dimensional case. Then, Séverac and Serre [38] proposed the following definition which has been used here:

$$
\nu \Delta_{S V V} v_{N}=\nu \Delta v_{N}+\nabla \cdot\left(\varepsilon_{N} Q_{N}\left(\nabla v_{N}\right)\right)
$$

where $\nabla v_{N}$ is the Jacobian of the vectorial function $v_{N}, \varepsilon_{N} Q_{N} \equiv \varepsilon_{N i}^{i} Q_{N i}^{i}$ with $i=1,2,3$ (corresponding to the $r, \theta, z$, directions respectively), and where $\varepsilon_{N i}^{i}, Q_{N i}^{i}$ are the maximum of viscosity and the $1 \mathrm{D}$ viscosity operator acting in direction $i$, respectively.

\section{Computational details}

The initial condition corresponds to a fluid at rest. No-slip boundary conditions are applied to all walls. There, $V_{r}=V_{z}=0$ on all walls, whereas $V_{\theta}$ is fixed at zero on the stator and the shroud and at the local disk velocity $\Omega r$ on the rotor and the hub. At the junctions rotor-stator, the tangential velocity component has been regularized by using a boundary function $V_{\theta}=e^{-(z-1) / \mu}$, with $\mu=0.006$ an arbitrary shape parameter independent of the grid size [36]. This function provides a reasonable representation of experimental conditions, while retaining spectral accuracy. It corresponds indeed to the radial and axial gaps already mentioned in Section II: $\delta_{1} / b=0.0036$ and $\delta_{2} / h=0.005$ respectively. In Taylor-Couette flow problems, Tavener et al. [41] mentioned that the effects of a clearance $\delta$ between the rotating disk and the stationary casing on the flow patterns away from the corners are negligible if $\delta$ remains sufficiently small: $\delta / b<0.02$, which is the case for both the experiment and the numerical simulation.

As shown previously, the SVV operator is parametrized in each direction by $\left(w_{T}, \epsilon_{N}\right)$. 
According to the theoretical results obtained by Tadmor [40], good values of such parameters are $w_{T} \approx O(\sqrt{N})$ and $\epsilon_{N} \approx O(1 / N)$, where $N$ is the degree of approximation in each direction. These values have also provided a good compromise between stability and accuracy in former numerical studies $[15,24]$. Let's notice that SVV operator affects at most the two-third of the spectrum on the highest frequencies $\left(w_{T}=0\right)$ and consequently, that DNS results are easily recovered for laminar flows, contrarily to some classical LES techniques as for example with the well-known spectral eddy viscosity model of Kraichnan [16]. The values of $w_{T}$ and $\epsilon_{N}$ used in the present LES are given in Table I.

Table I also presents grids and time steps used in the present study for the three Reynolds numbers. At $R e=10^{6}$, the calculations have been performed on the half cavity $([0, \pi])$ only. Indeed, due to the required resolution in the tangential direction, comparisons with experimental measurements have shown that this configuration offered the best arrangement between accuracy and confinement regard.

At the highest Reynolds number $R e=10^{6}$, accuracy in the description of both boundary layers has been checked by calculating the wall normal coordinate $z^{+}=z_{1} v_{\tau} / \nu$, where $z_{1}$ is the size of the smallest cell in the axial direction and $v_{\tau}$ is the total friction velocity defined as $v_{\tau}=\left[\nu^{2}\left(\frac{\partial V_{r}}{\partial z}\right)^{2}+\nu^{2}\left(\frac{\partial V_{\theta}}{\partial z}\right)^{2}\right]^{1 / 4}$. The universal value of the viscous sublayer thickness being close to 5 , if we want at least 5 collocation points to describe accurately this sublayer, $z^{+}$ needs to be close to 1 . Figure 2 presents the radial distribution of the axial wall coordinate near the walls. Typically, $z_{1} / h$ is equal to $3.76 \times 10^{-4}$. As $v_{\tau}$ increases towards the periphery of the cavity, $z^{+}$is an increasing function of the local radius. On both layers, $z^{+}$remains below or around 1 apart close to the shroud on the rotor side where $z^{+}$tends to 1.4 .

\section{MEAN FIELDS AND TURBULENCE STATISTICS}

The mean and turbulent quantities are presented in this Section for three values of the Reynolds number $R e$ in the range $\left[10^{5}-10^{6}\right]$. The calculated quantities have been averaged both in time (considering 26 disk revolutions for $R e=10^{5}$ up to 48 disk revolutions for $R e=10^{6}$ ) and in the tangential direction. Some of the results are compared with velocity measurements using a 2D LDV system. We define the dimensionless radial $r^{*}$ and axial $z^{*}$ coordinates as: $r^{*}=(r-a) /(b-a)$ and $z^{*}=z / h$. 


\section{A. Mean fields}

The axial profiles of the mean radial $V_{r}^{*}$ and tangential $V_{\theta}^{*}$ velocity components at midradius $\left(r^{*}=0.5\right)$ are shown for three Reynolds numbers in Figure 3. As the axial mean velocity component is everywhere very small compared to the two other components, it will not be discussed here. Whatever the Reynolds number considered in this study, the mean flows (Figure 3) correspond to separated boundary layer flows, belonging to the regime IV denoted by Daily and Nece [7]. The fluid near the rotating disk is driven away from the rotation axis $\left(V_{r}^{*}>0\right)$ due to centrifugal acceleration and this radial outflow along the rotor is compensated by flow directed towards the inner cylinder $\left(V_{r}^{*}<0\right)$ at the stator side. By analogy with the single disk problem, the boundary layer close to the rotor is known as the Ekman layer (although Ekman layer solutions are linear, one retains this terminology in the nonlinear case) whereas the boundary layer close to the stator is called the Bödewadt layer. These two boundary layers are separated by a nearly homogeneous core region, characterized by a quasi zero radial velocity $\left(V_{r}^{*} \simeq 0\right)$ and by a constant tangential velocity $V_{\theta}^{*}=K$, where $K$ is called the entrainment coefficient. As in laminar regimes [35], there exists on average, a main flow in the tangential direction coupled with a secondary flow in the meridional plane.

It is noteworthy that the entrainment coefficient $K$ is consistently below 0.5 midway between the disk which is the value obtained for the plane Couette flow. Its value at midradius $r^{*}=0.5$ (Table II) increases with the Reynolds number from 0.35 for $R e=10^{5}$ to 0.38 for $R e=10^{6}$ in the calculations and from 0.35 to 0.41 in the experiments. These values can be compared to the value $K \simeq 0.35$ measured by Cheah et al. [4] for $R e_{r}$ up to $2.6 \times 10^{5}$ and to the one $K=0.40$ obtained by Andersson and Lygren [1] at $R e=6.4 \times 10^{5}(G=0.1)$. Nevertheless, they remain substantially smaller than the semi-empirical value $K=0.43$ of Poncet et al. [26] for highly turbulent enclosed rotor-stator flows at $R e \geq 10^{6}$.

By comparing the $V_{r}$-profiles (Fig.3), the thickness of the Ekman boundary layer $\delta_{E}$, which is known to behave as $\sqrt{\nu / \Omega}$, decreases with $R e$ by about a factor two between $R e=10^{5}$ and $R e=10^{6}$ (Table II). This is characteristic of a rotating boundary layer which becomes turbulent. By conservation of mass, as there is no radial flow in the core, the Bödewadt boundary layer thickness $\delta_{B}$ behaves like the Ekman one. All the main results are summed up in Table II. The Bödewadt layer is almost two times thicker than the Ekman layer, which is itself about 10 times thicker than the thickness of the boundary layer over a 
free rotating $\operatorname{disk} \delta / h=\sqrt{\nu / \Omega h^{2}}$.

It is encouraging to observe from Figure 3 that the agreement between the numerical results and the velocity measurements is very satisfactory for the mean field. The both boundary layers along the rotor and the stator are well described by the LES, which captures the main features of rotor-stator flows. The largest differences with the LDV measurements are observed for $R e=10^{6}$. The calculations underestimate the measures of the $K$ coefficient of about 7\%, as previously observed by Andersson and Lygren [1] in a "wide-gap" cavity. The radial velocity component maxima on the rotor side is overestimated of about $14 \%$ at mid-radius. It appears from these remarks that LES calculations at $R e=10^{6}$ are certainly too dissipative at this location. A better agreement has been obtained at other radial locations as it is shown in the next Section. Some discrepancies on the velocity maxima can be also attributed to the size (in the axial direction) of the LDV probe volume, which becomes not negligible compared to the boundary layer thicknesses when Reynolds number increases. This leads indeed to averaged values in space only.

Additional characteristics of the mean flows are provided by a polar plot of the tangential and radial velocity components in the whole gap between the disks at $r^{*}=0.5$ (Figure 4). Whatever the Reynolds number considered, the polar profile is located between the similarity solution of Von Kármán [43] and the DNS calculations of Lygren and Andersson [21] performed at $R e=4 \times 10^{5}$. By increasing the Reynolds number, the LES polar plot goes away from the laminar solution. Whatever the value of $R e$, the polar plot corresponding to the stator boundary layer $\left(V_{r}<0\right)$ largely differs from the laminar flow, that confirms the turbulence feature of this boundary layer. Let's notice that at $R e=4 \times 10^{5}$ the LES profile almost matches the DNS profile. On the contrary, on the rotor side $\left(V_{r}>0\right)$, the polar plot is globally closer to the Von Kármán profile even if it goes away when Re increases. Such behavior suggests a transition to a turbulent regime from about $R e=4 \times 10^{5}$ that corresponds to a local Reynolds number at mid-radius of about $R e_{r}^{1 / 2}=407$. This is in agreement with the experimental and theoretical results of Lingwood [18]. The large difference observed with the profile of Lygren and Andersson [21] is then surprising and cannot be only explained by finite cavity effects.

Figures 5a to 5c show respectively the axial variations of $V_{r}^{+}, V_{\theta}^{+}$and $\left|V^{+}\right|=\left(V_{r}^{+2}+\right.$ $\left.V_{\theta}^{+2}\right)^{1 / 2}$ in wall coordinate $z^{+}\left(z^{+}\right.$is the relative distance to the nearest disk) for $R e=10^{6}$ and $r^{*}=0.5$. These are respectively the two streamwise mean velocity components relative to 
the disk speed and the magnitude of the corresponding velocity vector. We have numerically verified that the axial velocity component $V_{z}^{+}$is fairly close to zero along the disks. The velocities have been normalized by the tangential friction velocity $v_{\tau_{\theta}}=\left(\nu \partial V_{\theta} / \partial z\right)^{1 / 2}$ to enable direct comparisons with the work of Lygren and Andersson [21]. The magnitude of the velocity vector (Fig.5c) follows rather closely the law of the wall as obtained by Lygren and Andersson [21] in an open rotor-stator cavity. The profiles along both disks are compared to the profile of a turbulent boundary layer on a flat plate obtained by Rotta [30]. The linear region $\left|V^{+}\right|=z^{+}$of the velocity profile, called the viscous sublayer, expands to $z^{+}=10$ near the rotor, whereas it is more reduced along the stator (about to $z^{+}=5$ ). Lygren and Andersson [21] obtained a linear profile up to $z^{+} \simeq 7$ on both disks. Further from the walls, we recover the "log-region" $V^{+}=a+b \times \log _{10}\left(z^{+}\right)$with two coefficients $a$ and $b$, which differ from the values $(a=5, b=5.62)$ obtained by Lygren and Andersson [21] along the rotor. Note that for Couette-Poiseuille turbulent flows, the law of the wall is very close to: $V^{+}=1.0857 \log _{10}\left(z^{+}\right)+5.5$ as shown by Nakabayshi etal. [23]. The fundamental study of Bradshaw [2] has revealed that rotation substantially affects turbulence by introducing an extra linear term, depending on the Monin-Obukhov coefficient, in the logarithmic law of the wall. By comparison with the turbulent boundary layer on a flat plate [30], Figure 5 c clearly shows that no buffer region, which is usually the region enclosed between the sublayer and the log-region, is obtained in the present because of rotation. Another effect of rotation is the reduction of the log-region extent. To enable direct comparisons with the results of Lygren and Andersson in their open cavity, the profiles of $V_{r}^{+}$and $V_{\theta}^{+}$are also presented in Figures $5 \mathrm{a}$ and $5 \mathrm{~b}$ respectively. The same remarks arise from the variations of $V_{\theta}^{+}$compared to the ones of $\left|V^{+}\right|$. By considering the profiles of $V_{r}^{+}$(Fig.5a), the locations of the maximum crossflow are in close agreement with the values obtained by Lygren and Andersson [21] at Reynolds number $R e=4 \times 10^{5}$ although as expected, the location of the peaks here is further out from the wall as the Reynolds number is larger. Near the rotor, the maximum crossflow is reached at $z^{+}=16\left(z^{+}=15\right.$ in [21]) while near the stator the maximum is located further from the disk at $z^{+}=25\left(z^{+}=22\right.$ in [21]). Thus, for this radial location $r^{*}=0.5$, the location of the peak in the crossflow is not modified by finite cavity effects. 


\section{B. Turbulent statistics}

All six Reynolds stress tensor components have been calculated. The axial distributions of the two main normal components are shown together with available experimental measurements at mid-radius on Figure 6. The normal component $R_{z z}^{*}$ as well as the three shear components being found more than one order smaller than the previous ones are presented all together in Figure 7 at $R e=10^{6}$ only.

For the three considered Reynolds numbers, the turbulence is mainly concentrated in the boundary layers. Apart from the $R_{z z}^{*}$ component, the other five components reach indeed their maxima in the boundary layers and decrease to a value one order below $\left(6 \times 10^{-3} \Omega r\right)$ in the core (Fig.7). On the contrary, in their open cavity, Lygren and Andersson [21] reported that, except from the tangential components of the Reynolds stress tensor, the other components reach a maximum at the edge of the boundary layers. On Figure 6, the turbulence statistics confirm that whatever the Reynolds number the Bödewadt layer is turbulent at mid-radius while the Ekman layer gets turbulent at about $R e=4.10^{5}$ but with a turbulence intensity larger than on the stator. The turbulence intensities (scaled on the local speed of the rotor) remain almost constant in turbulent boundary layers when increasing the Reynolds number. That means that the turbulence intensity is intrinsic to the geometrical characteristics of the cavity and not to the energy injected into the system. Whatever the Reynolds number, LES results and experimental measurements fit quite well even in the core region, where the turbulence intensity is weak. Let's notice that it is generally not the case with RANS modeling for which the predictions in this low-Reynolds number flow region far from the disks are not satisfactory [27]. The locations of the peaks near the walls are also well predicted by the LES. When increasing the Reynolds number, these maxima go closer to the disks. At $R e=10^{6}$, the tangential and radial turbulence intensities have maxima at the same location $z^{*}=0.9904$ near the stationary disk and at different locations near the rotating disk: $z^{*}=0.0138$ for the $R_{r r}^{*}$ component and $z^{*}=0.0096$ for the $R_{\theta \theta}^{*}$ component.

The maximum error occurs in the prediction of the peak values. The singular behavior observed at $R e=10^{5}$ on the rotor side can be explained by the inherent difficulties of the LDV system previously mentioned in Section II. At $R e=10^{6}$, the turbulence intensities are rather the same in both boundary layers by considering the experimental data, whereas the LES leads to a regime where the Ekman layer is slightly more turbulent than the Bödewadt 
layer by looking at the $R_{r r}^{*}$-profile. This feature has been formerly reported by Lygren and Andersson [21] when they compared their DNS results with the experiments of Itoh [13]. They attributed this discrepancy to the increased mixing between the boundary layers due to the presence of the shroud in the experiments of Itoh. The present results show that the presence of the shroud cannot explain this discrepancy, as LES and experiment used the same cavity model.

The general trend is that LES results overestimate the turbulence intensity in the tangential direction $R_{\theta \theta}^{*}$ while they underestimate it in the crossflow direction $R_{r r}^{*}$, particularly in the stator boundary layer. On Figure 6 and at $R e=10^{6}$, the numerical results predict a $R_{\theta \theta}^{*}$ component almost twice larger than the $R_{r r}^{*}$ component, whereas the experimental measurements give components of about the same order. That suggests that the turbulence anisotropy is stronger in LES than in experiments. There is no clear reason that explains such behavior if not the anisotropy of the grid computation, which is globally coarser in the radial direction than in two other ones. This reason was previously advocated by Scotti et al. [33] to explain some discrepancies between LES and experiments in wall-bounded flows. The grid is indeed non homogeneous and tighter close to the walls. For example, at mid-radius, the grid spacing is $0.052 h, 0.033 h$ and $0.019 h$ in the radial, tangential and axial directions respectively. This phenomenon could certainly be reduced with grid refinement in the radial and tangential directions. As a consequence, the predicted Reynolds stress tensor is more anisotropic than the measured one.

If we consider the shear components of the Reynolds stress tensor at $R e=10^{6}$ (Fig.7), we clearly see that the peaks of $R_{r \theta}^{*}$ are the largest in both boundary layers compared to the two other shear components. It is a major difference with two-dimensional boundary layer flows where this component is usually neglected. One major difference between the idealized flow considered by Lygren and Andersson [21] and the present study is that the $R_{r z}^{*}$ and $R_{\theta z}^{*}$ components are negligible in the present LES, whereas these authors obtained magnitudes of these two components quite comparable to the $R_{r \theta}^{*}$ component. Moreover, the $R_{r \theta}^{*}$ shear component is negative on both disks in the open cavity of [21], whereas the shear is found here to be positive on the rotor with a magnitude higher than on the stator side.

In order to show the turbulent flow regions in the meridional plane, the isolines of the turbulence kinetic energy $k^{*}=k /(\Omega b)^{2}$ are presented in Figure 8 at three Reynolds numbers. The maximum value of the turbulence kinetic energy evolves from $k_{\max }^{*}=4.7 \times 10^{-3}$ 
to $k_{\max }^{*}=9 \times 10^{-3}$ for increasing values of the Reynolds number, and its location moves from the stator boundary layer to the edge of the rotor layer. At $R e=10^{5}$, the turbulence is mainly confined in the boundary layers along the stator and the shroud. A small turbulent region is also observed at the impingement on the rotor of the flow coming from the stator and accelerated along the rotating hub. The maximum of $k^{*}$ is obtained at the junction between the stator and the shroud where the flow coming from the rotor impinges the stator. By increasing the Reynolds number up to $R e=4 \times 10^{5}$, the Ekman layer gets turbulent from about mid-radius to $r^{*}=1$ corresponding to the largest values of the local Reynolds number. The maximum of $k^{*}$ has moved to the bottom of the shroud and the hub is also now turublent. At $R e=10^{6}$, the map is relatively similar to the previous one at $R e=4 \times 10^{5}$. The maximum is nevertheless two times larger and has slightly moved on the left hand side along the rotor boundary layer.

\section{FINITE CAVITY EFFECTS}

Due to the presence of an inner and an outer cylinders at $r^{*}=0$ and $r^{*}=1$ respectively, the flow is radially confined. Then, finite cavity effects on the mean field and turbulence statistics have been explored at $R e=10^{6}$.

Axial profiles of the mean radial $V_{r}^{*}$ and tangential $V_{\theta}^{*}$ velocity components at four radial locations are shown on Figure 9. The same agreement observed at mid-radius (Fig.3) between experimental measurements and LES results is observed for the three over radial locations. In a flow region of radial extension $0.3 \leq r^{*} \leq 0.7$, a self-similar behavior is observed and the boundary layers remain separated (regime IV [7]). Close to the outer shroud $\left(r^{*}=0.9\right)$, the Bödewadt layer thickens and the flow in the core is clearly influenced by the boundary conditions. This effect on the flow in the core is besides larger on the LES results than on the experimental data. According to the mass flow conservation, the entrainment coefficient $\mathrm{K}$ increases with the radius from 0.35 at $r^{*}=0.3$ to 0.38 at $r^{*}=0.9$ whereas the maxima of $\left|V_{r}^{*}\right|$ decrease in both layers.

As the consequence, the flow along the disks is no longer parallel contrary to the flows in infinite cavities [21]. The radial evolutions of the thicknesses of both boundary layers are shown on Figure 10, together with the predictions of the RSM of Poncet [25] for the same Reynolds number $R e \simeq 10^{6}$ but in a cavity of aspect ratio about five times larger 
$\left(G=23.6, R_{m}=1.36\right)$. Except in the endwall regions, both numerical results give qualitatively a good agreement. On the rotor side, the boundary layer thickness increases with the radius whereas on the stator side the flow remains almost parallel outside the endwall regions $\left(0.1 \leq r^{*} \leq 0.7\right)$. In that zone, the averaged value of $\delta_{B} / h$ is 1.5 larger than the averaged value of the Ekman layer thickness: $\overline{\delta_{B}} / h \simeq 1.5 \overline{\delta_{E}} / h=0.061$. This result is close to the one obtained by Daily and Nece [7] for turbulent flows with separated boundary layers: $\delta_{B} \simeq 1.7 \delta_{E}$. Let's notice that this behavior seems different of the one observed in the laminar regime. Indeed, Gauthier et al. [9] reported a decreasing of $\delta_{B}$ for increasing radial locations, as $\delta_{B}=\delta\left(6.9-5.3 r^{*}\right)$, and an almost constant Ekman layer thickness $\delta_{E}=2.2 \delta$ far from the endwalls. The discrepancies between the LES and the RSM close to the cylinders is firstly attributed to the much larger value of $G$ considered by Poncet [25] that considerably diminishes the effects of the endwall layers. The difficulty of the RSM to take into account large recirculation zones as well as the two-dimensional hypothesis can be also evoked.

In the shroud boundary-layer, an intense shear is produced by the differential rotation between the core of the flow in solid body rotation $(K \simeq 0.4 \Omega)$ and the outer stationary cylinder. By analogy with the classical Taylor-Couette problem of the flow between differentially rotating cylinders or spheres, this internal shear layer is called the Stewartson layer [39]. The present cavity can be indeed considered as a rotor-stator Taylor-Couette system of very large aspect ratio with the flow confined between an inner rotating cylinder and an outer stationary cylinder. Figure 11 presents the axial variation of the Stewartson layer thickness $\delta_{s}$ for $R e=10^{6}$. The LES results can be scaled by a linear fit $\delta_{s} / b=17 \times z^{*}-0.076$ showing that the Stewartson layer is non homogeneous in the axial direction contrary to the classical Taylor-Couette problem. After the impingement of the outward radial flow along the rotor, the axial flow slows down along the shroud and as a consequence $\delta_{S}$ increases. At the edge of the rotor, the Ekman layer turns into the axial flow of the Stewartson layer in a region of which both the radial and axial dimensions are $O\left(R e^{-1 / 2}\right)$ [42]. In the present case, the connection between the two boundary layers occurs when both dimensions are $1.62 \times R e^{-1 / 2}$, which corresponds to $\delta_{s} / b=0.0115$ on Figure 11 and to $\delta_{E} / h=0.081$ on Figure 10. Along the stator, the thickening of the Stewartson layer is also closely linked to the thickening of the Bödewadt layer at the periphery of the cavity.

Finite cavity effects also influence the turbulence statistics as shown on Figure 12. What- 
ever the radial location, the turbulence remains mainly confined in the boundary layers, whereas the inviscid core remains laminar. The turbulence intensities slightly increase towards the periphery of the cavity as expected by the increase of the local Reynolds number. At $r^{*}=0.3\left(R e_{r}=2 \times 10^{5}\right)$, the boundary layers are already turbulent with comparable turbulence intensities. At the periphery of the cavity $r^{*}=0.9\left(R e_{r}=8.62 \times 10^{5}\right)$, the Ekman layer becomes more turbulent than the Bödewadt layer. Note that, whatever the radial location, the $R_{\theta \theta}^{*}$ component remains almost twice larger than the $R_{r r}^{*}$ component along the disks. The agreement between the LES and the velocity measurements remains still satisfactory over the radius. Nevertheless, as already observed when varying the Reynolds number in section IV, the general trend of the LES is to underestimate the turbulence level in the crossflow direction and to overestimate it in the tangential direction in the whole cavity.

To investigate the effects on the flow structures arising from the three-dimensionality of the mean flow, anisotropy invariant maps are shown at four different radial locations and $R e=10^{6}$ (Figure 13). The second $A_{2}$ and third $A_{3}$ invariants of the anisotropy tensor $a_{i j}$ of the second moments of the fluctuations are defined as:

$$
A_{2}=a_{i j} a_{j i}, A_{3}=a_{i j} a_{j k} a_{k i}
$$

where $a_{i j}=R_{i j} / k-\frac{2}{3} \delta_{i j}[20],\left(\delta_{i j}\right.$ the Kronecker symbol). Thus, the anisotropy invariant maps do not provide spatial informations on the flow structures but crucial informations on the structure properties and especially on their velocity fluctuations. Let's notice that, whatever the radial location, the LES results respect the realizability diagram of Lumley [20] as they remain within the region delimited by the three solid lines. Very close to the disks, the turbulence tends to follow the two-component behavior $\left(A_{3}=A_{2}-8 / 9\right)$ as the wall normal fluctuations are damped more effectively than fluctuations parallel to the disks. Whatever the radial location, the turbulence is fairly close to the isotropic case $\left(A_{2}=A_{3}=0\right)$ in the core of the flow. The domination of the $R_{\theta \theta}^{*}$ component (Fig.7) as well as the positive sign of the third invariant $A_{3}$ of the anisotropy tensor in the Bödewadt layer suggest a "cigar-shaped" structuring of turbulence in the tangential direction. This kind of structuring is well known in dominantly rotating turbulence [3]. At the edge of the Ekman layer $\left(z^{*} \simeq 0.05\right)$, for $0.3 \leq r^{*} \leq 0.7$, the third invariant gets negative and close to the two-dimensional isotropic turbulence limit $(-2 / 9,2 / 3)$. In that region, the levels of the two normal components $R_{\theta \theta}^{*}$ and $R_{r r}^{*}$ are quite comparable as it can be seen on Figure 7 . This 
is an indicator of "pancake" structuring contained in planes perpendicular to the rotation axis. One interesting feature is that closer to the shroud $\left(r^{*}=0.9\right)$ where high turbulence level prevails, these vortex disappear, which is certainly due to the confinement.

\section{THREE-DIMENSIONAL BOUNDARY LAYERS}

The flow between rotating disks is one of the simplest case where the boundary layers are three-dimensional from their inception. In a classical way, a three-dimensional turbulent boundary layer (3DTBL) is a boundary layer where:

(i) the direction of the mean velocity vector is non-constant with respect to the distance from the wall,

(ii) the direction of the Reynolds shear stress vector in planes parallel with the wall is not aligned with the mean velocity gradient vector,

(iii) the value of the Townsend structural parameter $a_{1}=\tau / 2 k$, defined as the ratio of the shear stress vector magnitude $\tau=\left({\overline{v_{\theta}^{\prime} v_{z}^{\prime}}}^{2}+{\overline{v_{r}^{\prime} v_{z}^{\prime}}}^{2}\right)^{1 / 2}$ to twice the turbulence kinetic energy $k$ is lower than the typical limit for two-dimensional turbulent boundary layers (2DTBL): $a_{1}=0.15$.

The 3DTBL behaviors of the rotor and stator boundary layers are here investigated at $R e=10^{6}$. Figure 14 shows the axial variations of the mean velocity angle $\gamma_{m}=$ $\arctan \left(V_{r} / V_{\theta}\right)$ in the two boundary layers at $r^{*}=0.3$. On the rotor side, the angle increases from zero at the rotating disk to $16.5^{\circ}$ at $z^{+}=25$, then slightly decreases to about $-0.5^{\circ}$ outside the Ekman layer. On the stator side, the angle monotonically decreases from zero to $-34^{\circ}$. Let's notice that the same behaviors have been obtained for $0.3 \leq r^{*} \leq 0.9$. This checks the condition (i) given above for a large radial extent domain $0.3 \leq r^{*} \leq 09$.

The misalignment between the direction of the Reynolds shear stress vector in planes parallel with the wall and the mean velocity gradient vector (condition (ii)) is observed in the present LES on both disks. In Figure 14, the axial variations of two characteristic angles, the mean gradient velocity angle $\gamma_{g}=\arctan \left(\frac{\partial V_{r} / \partial z}{\partial V_{\theta} / \partial z}\right)$ and the turbulent shear stress angle $\gamma_{\tau}=\arctan \left(\overline{v_{r}^{\prime} v_{z}^{\prime}} / \overline{v_{\theta}^{\prime} v_{z}^{\prime}}\right)$ are shown. The lag between $\gamma_{\tau}$ and $\gamma_{g}$ is large towards the boundary layers with a maximum value about $94^{\circ}$ on the rotor at $r^{*}=0.3$ to be compared 
with the value $18^{\circ}$ reported by Lygren and Andersson [21] in infinite disk system. Note that this lag increases up to $151^{\circ}$ at $r^{*}=0.9$ (not presented here). In their numerical study of non-stationary 3DTBL, Coleman et al. [5] obtained large values of the lag especially near the wall and inferred it from the slow growth of the spanwise component of the shear stress. These authors observed also the change of the sign of the gradient angle $\gamma_{g}$. Such large values of this lag make the assumption of eddy-viscosity isotropy to fail for the prediction of such flows.

Compared to a two-dimensional turbulent boundary layer (2DTBL) as the one found in a Couette flow, one characteristic of a 3DTBL is the relative reduction of the magnitude of the shear-stress vector in planes parallel with the disks with the turbulence kinetic energy $k$. This measure is done using the Townsend structural parameter $a_{1}=\tau / 2 k$. Typically $a_{1}=0.15$ for a wide range of 2DTBLs. The variations of $a_{1}$ have been reported in Figure 15 at four radial locations in both the Ekman and Bödewadt layers, whose thicknesses depend on the local radius. Whatever the considered locations, the maximum and minimum peak values of $a_{1}$ are 0.062 and 0.038 , respectively. They are reached on the stator side and are significantly reduced below the limiting value 0.15 for a 2DTBL (condtion (iii) verified). This behavior is similar to this reported by Itoh et al. [12] and Littell and Eaton [19] from their measurements and suggests the three-dimensional turbulent nature of the flow along the rotor and stator walls. Let's notice that the range of values of $a_{1}$ is the same on both layers. This reduction of $a_{1}$ indicates also that the shear stress in this type of flow is less efficient in extracting turbulence energy from the mean field. Note that $a_{1}>0.15$ is obtained only very locally on the inner and outer cylinders. These values are much smaller than those found by Andersson and Lygren [1] in the open cavity, suggesting that finite cavity effects increase the three-dimensionality of the mean flow.

\section{FLOW STRUCTURES}

The flow structures in the disks boundary layers evolve from spiral arms to annuli when one increases the rotation rate of the disk. The transition to turbulence for separated boundary layer flows has been widely addressed experimentally $[25,32]$ and numerically [35]. During the transition process in the laminar regime, the flow structures evolve from circular to spiral rolls. 
Some flow visualizations have been firstly performed from above the stator for two Reynolds numbers $R e=4.1 \times 10^{4}$ and $R e=10^{5}$ using a CCD video camera. The cavity has been filled up with water and seeded with reflective particles of kalliroscope $(30 \times 6 \times 0.07 \mu \mathrm{m})$. For $R e=4.1 \times 10^{4}$ (Fig.16a), the flow structure is already complex but it is still organized by large structures showing spiral patterns with defects. For $R e=10^{5}$, the Bödewadt layer is now clearly turbulent (Fig.16b). The flow structures are much thinner and more aligned on the tangential direction.

To identify numerically these coherent vortices, the Q-criterion of Hunt et al. [10] is used. It defines a vortex as a spatial region where the Euclidean norm of the vorticity tensor $\boldsymbol{\Omega}=\frac{1}{2}\left[\nabla \mathbf{v}-(\nabla \mathbf{v})^{T}\right]$ dominates that of the rate strain $\mathbf{S}=\frac{1}{2}\left[\nabla \mathbf{v}+(\nabla \mathbf{v})^{T}\right]$ : $Q=\frac{1}{2}\left[|\boldsymbol{\Omega}|^{2}-|\mathbf{S}|^{2}\right]>0$. Figure 17 presents the isosurface of the Q-criterion in both boundary layers and for the three Reynolds numbers under consideration. At $R e=10^{5}$, only the stator boundary layer (Fig.17b) is turbulent with increasing intensities towards the periphery. On the rotor side, turbulent flow regions are only confined close to the junctions with the hub and the shroud where the flow strongly impacts the wall. The inner rotating hub is found to have a strong destabilizing effect, accelerating the flow and strengthening the vortices coming from the Bödewadt layer to the Ekman layer. It is contrary to the configuration where the hub is stationary [34]. For $R e=4 \times 10^{5}$, the flow along the stator is fully turbulent as expected, while the rotor layer is now transitional turbulent. About 19 spiral arms forming a positive angle $\epsilon \simeq 16^{\circ}$ with the tangential direction (as they roll up in the rotation sense) appear in the Ekman layer for $0.14 \leq r^{*} \leq 0.61$ (Fig.17c) where the flow is laminar unstable $\left(89 \leq R e_{r}^{1 / 2} \leq 386<500\right)$. These structures are characteristic of the Type I instability (crossflow instability), which plays an important role in the transition process to turbulence. These results are consistent with previous results (see the review of Saric et al. [31]). Close to the outer radius, the structures are thinner and more axisymmetric, which is characteristic of a turbulent flow. At $R e=10^{6}$ (Fig.17e,f), the flow gets fully turbulent in both boundary layers. Along the stator, as expected, turbulence intensities increase for increasing values of the local Reynolds number and the coherent vortical structures, which are aligned with the tangential direction, get thinner. Let's notice that, for all considered Reynolds numbers, any three-dimensional vortical structures were observed here in the core of the flow, contrary to the recent experimental observations of Czarny et al. [6]. 


\section{CONCLUSION}

Turbulent flows in an enclosed rotor-stator cavity have been investigated up to $R e=10^{6}$ both numerically using high-order LES and experimentally using LDV measurements. As far as the authors are aware, efficient LES of fully turbulent flow in an enclosed rotor-stator cavity have not been performed before. The disks are enclosed by an inner hub attached to the rotor and an outer shroud attached to stator involving finite cavity effects that were not taken into account in former LES results of Lygren and Andersson [1, 21, 22].

The LES has been performed here using a spectral vanishing viscosity method providing solutions that converge to solutions of Navier-Stokes equations when the cutoff goes to infinity, and preserving the spectral accuracy of smooth solutions. Such flows are difficult to compute when using spectrally accurate numerical schemes, that results directly from the fact that spectral approximations are much less diffusive than low order ones.

Numerical results and experimental measurements have been presented for three Reynolds numbers, $R e=10^{5}, R e=4 \times 10^{5}$ and $R e=10^{6}$ in order to show the increasing complexity in modeling these flows when rotation is increased. The boundary layers are separated with a turbulent Ekman layer on the rotor from $R e=4 \times 10^{5}$ and a Bödewadt layer on the stator already turbulent at $R e=10^{5}$. On both layers, finite cavity effects have been shown at $R e=10^{6}$ with a radial dependence of the main features of the turbulent flow. The turbulence intensities slightly increase going towards the periphery. In the flow regions where the turbulence level is the weakest, the Q-criterion reveals spiral arm patterns related to the crossflow instability. In the fully turbulent flow regions, the structures become much thinner and aligned on the tangential direction.

At $R e=10^{6}$, the anisotropy invariant map reveals some features related to "cigar-shaped vortex" aligned on the tangential direction on the rotor side and related to "pancake-shaped vortex" on the stator side. The reduction of the Townsend structural parameter $a_{1}$ under the typical limit 0.15 , as well as the strong misalignment between the shear stress vector and the mean velocity gradient vector, highlight the three-dimensional nature of both boundary layers with a degree of three-dimensionality much higher than in the idealized system studied by Lygren and Andersson [1, 21].

The LES results compare very favorably with the LDV measurements that is very encouraging for this numerical approach to deal with complex flows. Improvements in the 
future should come from a coupling of the SVV procedure to the flow dynamics in order to optimize the dissipation of the model. The viscosity kernel parameters could be selected adaptively by relating them to the dynamics of the flow, i.e. the strain field.

\section{Acknowledgments}

The authors acknowledge the IDRIS (CNRS, Orsay, France) computing center where the computations have been carried out on the NEC SX-5 supercomputer (program 060242). The authors are very grateful to Roland Schiestel (CNRS / IRPHE) and Professors R. Pasquetti (CNRS / Lab. J. Dieudonné, Nice) and B.E. Launder (Univ. of Manchester) for fruitful discussions. The work was supported by CNRS in the frame of DFG-CNRS program "LES of complex flows". Support for E. Séverac by a CNRS grant is also acknowledged.

[1] H.I. Andersson and M. Lygren. LES of open rotor-stator flow. Int. J. Heat Fluid Flow, $27(4): 551-557,2006$.

[2] P. Bradshaw. Effects of streamline curvature on turbulence. AGARDograph., 169, 1973.

[3] C. Cambon. Turbulence and vortex structures in rotating and stratified flows. Eur. J. Mech. B/Fluids, 20:489-510, 2001.

[4] S.C. Cheah, H. Iacovides, D.C. Jackson, H. Ji, and B.E. Launder. Experimental investigation of enclosed rotor-stator disk flows. Exp. Therm. Fluid Sci., 9:445-455, 1994.

[5] G.N. Coleman, J. Kim, P.R. Spalart. A numerical study of strained three-dimensional wallbounded turbulence. J. Fluid Mech., 416:75-116, 2000.

[6] O. Czarny, H. Iacovides and B.E. Launder. Precessing Vortex Structures in Turbulent Flow within Rotor-Stator Disc Cavities. Flow, Turbulence and Combustion, 69:51-61, 2002.

[7] J.W. Daily and R.E. Nece. Chamber dimension effects on induced flow and frictional resistance of enclosed rotating disks. ASME J. Basic Eng., 82:217-232, 1960.

[8] L. Elena and R. Schiestel. Turbulence modeling of rotating confined flows. Int. J. Heat Fluid Flow, 17:283-289, 1996.

[9] G. Gauthier, P. Gondret and M. Rabaud Axisymmetric propagating vortices in the flow between a stationary and a rotating disk enclosed by a cylinder. J. Fluid Mech., 386:105-126, 
1999.

[10] J.C.R. Hunt, A. Wray, and P. Moin. Eddies, streams, and convergence zones in turbulent flows. Proc. 1988 Summer program, NASA Stanford Center for Turbulence Research, Report CTR-S88: 193-208, 1988.

[11] M. Itoh, Y. Yamada, S. Imao, and M. Gonda. Experiments on turbulent flow due to an enclosed rotating disk. In W. Rodi and E.N. Ganic, editors, Engineering Turbulence Modeling and Experiments, pages 659-668, New-York, 1990. Elsevier.

[12] M. Itoh, Y. Yamada, S. Imao, and M. Gonda. Experiments on turbulent flow due to an enclosed rotating disk. Exp. Thermal Fluid Sci., 5:359-368, 1992.

[13] M. Itoh. Experiments on the turbulent flow in the narrow clearance between a rotating and a stationary disk. Ed. B.F. Carroll, T. Kobayashi and M.J. Morris, Turbulent flows, ASMEFED, vol.208, pages 27-32, 1995.

[14] H.S. Kang, H. Choi, and J.Y. Yoo. On the modification of the near-wall coherent structure in a three-dimensional turbulent boundary layer on a free rotating disk. Phys. Fluids, 10(9):23152322, 1998.

[15] G.S. Karamanos and G.E. Karniadakis. A spectral vanishing viscosity method for large eddy simulation. J. Comp. Phys., 163:22-50, 2000.

[16] R.H. Kraichnan Eddy viscosity in two and three dimensions, J. Atmos. Sci. 33: 1521, 1976.

[17] B.E. Launder and D.P. Tselepidakis. Application of a new second moment closure to turbulent channel flow rotating in orthogonal mode. Int. J. Heat Fluid Flow, 15: 2, 1994.

[18] R.J. Lingwood. Absolute instability of the Ekman layer and related rotating flows. J. Fluid. Mech., 331:405-428, 1997.

[19] H.S. Littell and J.K. Eaton. Turbulence characteristics of the boundary layer on a rotating disk. J. Fluid. Mech., 266:175-207, 1994.

[20] J.L. Lumley. Computational modeling of turbulent flows. Adv. Appl. Mech., 18:123-176, 1978.

[21] M. Lygren and H.I. Andersson. Turbulent flow between a rotating and a stationary disk. J. Fluid. Mech., 426:297-326, 2001.

[22] M. Lygren and H.I. Andersson. Large eddy simulations of the turbulent flow between a rotating and a stationary disk. Z. Angew. Math. Phys., 55:268-281, 2004.

[23] K. Nakabayshi, O. Kitoh, and Y. Katoh. Similarity laws of velocity profiles and turbulence characteristics of Couette-Poiseuille turbulent flows. J. Fluid Mech., 507:43-69, 2004. 
[24] R. Pasquetti and C.J. Xu. High-order algorithms for large-eddy simulation of incompressible flows. J. Sci. Comp., 17(1-4):273-284, 2002.

[25] S. Poncet. Écoulements de type rotor-stator soumis à un flux axial: de Batchelor à Stewartson. PhD thesis, Université Aix-Marseille I, 2005.

[26] S. Poncet, M.P. Chauve, and P. Le Gal. Turbulent rotating disk with inward throughflow. J. Fluid. Mech., 522:253-262, 2005.

[27] S. Poncet, M.P. Chauve, and R. Schiestel. Batchelor versus stewartson flow structures in a rotor-stator cavity with throughflow. Phys. Fluids, 17(7), 2005.

[28] A. Randriamampianina and S. Poncet. Turbulence characteristics of the Bödewadt layer in a large enclosed rotor-stator system. Phys. Fluids, 18: 055104, 2006.

[29] I. Raspo, S. Hugues, E. Serre, A. Randriamampianina, and P. Bontoux. A spectral projection method for the simulation of complex three-dimensional rotating flows. Computers and Fluids, 31:745-767, 2002.

[30] J.C. Rotta. Turbulente Strömungen. Teubner, Stuttgart, 1972.

[31] W.S. Saric, H.L. Reed, and E.B. White Stability and transition of three-dimensional boundary layers. Ann. Rev. Fluid Mech., 35:413-440, 2003.

[32] L. Schouveiler, P. Le Gal and M.P. Chauve. Instabilities of the flow between a rotating and a stationary disk. J. Fluid Mech., 443:329-350, 2001.

[33] A. Scotti, C. Meneveau and D.K. Lilly. Generalized Smagorinsky model for anisotropic grids. Phys. Fluids, A5:2306, 1993.

[34] E. Serre, P. Bontoux and B.E. Launder. Direct Numerical Simulation of transitional turbulent flow in an enclosed rotor-stator cavity. Flow, Turbulence \& Combustion, 69:35-50, 2002.

[35] E. Serre, E. Crespo del Arco and P. Bontoux. Annular and spiral patterns in flows between rotating and stationary discs. J. Fluid Mech., 434:65-100, 2001.

[36] E. Serre and J. P. Pulicani. 3D pseudo-spectral method for convection in rotating cylinder. Int. J. Comp. Fluids, 30(4):491-519, 2001.

[37] E. Séverac, E. Serre, R. Pasquetti and B.E. Launder, A stabilization technique to study turbulent rotating flows using high-order numerical method, In Advances in Turbulence X, 861, CIMNE, Eds. H.I. Andersson, P.A. Krogstad, Barcelona 2004.

[38] E. Séverac and E. Serre. A spectral vanishing viscosity LES model for the simulation of turbulent flows within rotating cavities. accepted for publication in J. Comp. Phys., 2006. 
[39] K. Stewartson. On almost rigid rotations. J. Fluid Mech., 26:17-26, 1957.

[40] E. Tadmor. Convergence of spectral methods for nonlinear conservation laws. SIAM J. Numer. Anal., 26(1):30-44, 1989.

[41] S.J. Tavener, T. Mullin, and K.A. Cliffe. Novel bifurcation phenomena in a rotating annulus. J. Fluid. Mech., 229:483-497, 1991.

[42] A.I. Van de Vooren. The connection between Ekman and Stewartson layers for a rotating disk. J. Eng. Math., 27:189-207, 1993.

[43] T. Von Kármán. Uber laminare und turbulente Reibung. Z. Angew. Math. Mech., 1:233-252, 1921.

[44] X. Wu and K.D. Squires. Prediction and investigation of the turbulent flow over a rotating disk. J. Fluid. Mech., 418:231-264, 2000. 


\begin{tabular}{c|c|c|c|c}
$R e$ & $w_{T}(r, \theta, z)$ & $\epsilon_{N}$ & $\operatorname{grid}$ & $\delta t$ \\
\hline $10^{5}$ & $3 N / 4, N / 2, \sqrt{N}$ & $1 /(2 N), 1 /(2 N), 1 /(2 N)$ & $81,150,49$ & $10^{-4}$ \\
\hline $4 \times 10^{5}$ & $0.8 \sqrt{N}, \sqrt{N}, \sqrt{N}$ & $1 /(2 N), 1 /(2 N), 1 /(2 N)$ & $121,181,65$ & $10^{-4}$ \\
\hline $10^{6}$ & $2 \sqrt{N}, 5 \sqrt{N}, 4 \sqrt{N}$ & $1 / N, 1 / N, 1 / N$ & $151,241,81$ & $10^{-5}$
\end{tabular}

Table I: Computational parameters. 


\begin{tabular}{c|c|c|c|c} 
Reynolds number & $K$ & $\delta_{E} / h$ & $\delta_{B} / h$ & $\delta / h$ \\
\hline $10^{5}$ & 0.35 & 0.104 & 0.222 & 0.022 \\
\hline $4 \times 10^{5}$ & 0.36 & 0.071 & 0.147 & 0.011 \\
\hline $10^{6}$ & 0.38 & 0.055 & 0.084 & 0.007
\end{tabular}

Table II: Influence of the Reynolds number on the entrainment coefficient $K$ and on the thicknesses of the boundary layers for $r^{*}=0.5$ (LES). 
- Fig.1: Schematic representation of the cavity with relevant notation.

- Fig.2: Radial evolution of the wall normal axial coordinate $z^{+}$near the rotor and the stator sides for $R e=10^{6}$ (half cavity).

- Fig.3: Axial profiles of the mean radial $V_{r}^{*}$ and tangential $V_{\theta}^{*}$ velocity components at $r^{*}=0.5$ for three Reynolds numbers. Comparison between $(-)$ the LES results and (o) the LDV data.

- Fig.4: Polar plot of the velocity distribution in the whole gap between the disks at $r^{*}=0.5$ for three Reynolds numbers. Comparison between $(-)$ the LES results, (o) the LDV data, (-.) the laminar Von Kármán [43] solution and (--) the DNS results of Lygren and Andersson [21].

- Fig.5: Mean (a) radial $V_{r}^{+}$and (b) tangential $V_{\theta}^{+}$velocity components in wall units near the rotor (solid lines) and the stator (dashed lines) normalized with the tangential friction velocity for $R e=10^{6}$ at $r^{*}=0.5$. (c) Magnitude of the corresponding velocity vector $\left|V^{+}\right|$near the rotor (solid lines) and the stator (dashed lines) compared to the turbulent boundary layer over a flat plate (dash-dot line) by Rotta [30].

- Fig.6: Axial profiles of the radial $R_{r r}^{*}$ and tangential $R_{\theta \theta}^{*}$ Reynolds stress tensor components at $r^{*}=0.5$ for three Reynolds numbers. The square roots of the Reynolds stresses have been normalized by the local speed of the disk. Comparison between (-) the LES results and (o) the LDV data.

- Fig.7: Axial variations of the Reynolds stress tensor components for $r^{*}=0.5$ and $R e=10^{6}$.

- Fig.8: Isolines of the turbulence kinetic energy $k^{*}=k /(\Omega b)^{2}$ at: (a) $R e=10^{5}$, $k^{*} \leq 4.6 \times 10^{-3}$, (b) $R e=4 \times 10^{5}, k^{*} \leq 7 \times 10^{-3}$, (c) $R e=10^{6}, k^{*} \leq 6 \times 10^{-3}$ (LES).

- Fig.9: Axial profiles of the mean radial $V_{r}^{*}$ and tangential $V_{\theta}^{*}$ velocity components for $R e=10^{6}$ at four radial locations. Comparison between (-) the LES results and (o) the LDV data.

- Fig.10: Radial distributions of the boundary layer thicknesses: (a) Ekman boundary layer thickness $\delta_{E} / h$, (b) Bödewadt boundary layer thickness $\delta_{B} / h$. Comparisons 
between (-) the present LES $\left(R e=10^{6}, G=5\right)$ and $(--)$ the predictions of the Reynolds Stress Model of Poncet [25] $\left(R e=1.04 \times 10^{6}, G=23.6\right)$.

- Fig.11: Axial distribution of the Stewartson boundary layer thickness $\delta_{S} / b$ along the external cylinder for $R e=10^{6}$. Comparison between $(\times)$ the LES results and $(-)$ a linear interpolation.

- Fig.12: Axial profiles of the radial $R_{r r}^{*}$ and tangential $R_{\theta \theta}^{*}$ Reynolds stress tensor components for $R e=10^{6}$ at four radial locations. Comparison between $(-)$ the LES results and (o) the LDV data.

- Fig.13: Anisotropy invariant map for $R e=10^{6}$ at four radial locations. Dashed lines: LES, solid lines: realizability diagram of Lumley [20].

- Fig.14: Axial evolutions of the mean velocity angle $\gamma_{m}$, the mean gradient angle $\gamma_{g}$ and the shear stress angle $\gamma_{\tau}$ for $R e=10^{6}$ at $r^{*}=0.3$ : (a) along the rotor side, (b) along the stator side.

- Fig.15: Townsend structural parameter $a_{1}=\tau /(2 k)$ on both disks for $R e=10^{6}$.

- Fig.16: Flow visualizations from above the stator for (a) $R e=4.1 \times 10^{4}$, (b) $R e=10^{5}$.

- Fig.17: Isosurface plot (top view) of the Q-criterion in the rotor boundary layer (a,c,e) and in the stator boundary layer (b,d,f) for $R e=10^{5}(\mathrm{a}, \mathrm{b}), R e=4 \times 10^{5}(\mathrm{c}, \mathrm{d})$ and $R e=10^{6}(\mathrm{e}, \mathrm{f})$. Note that the disk rotates counterclockwise. 


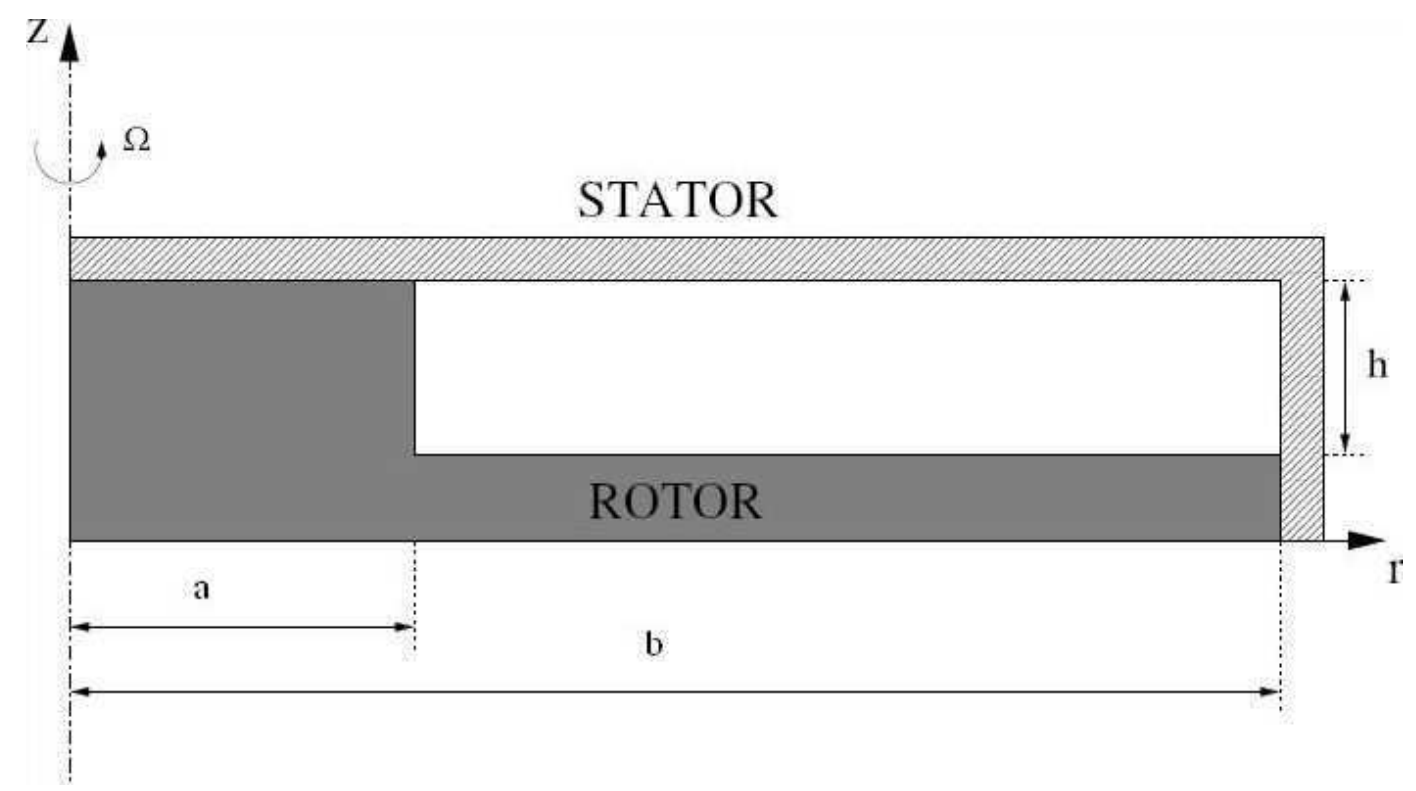

Figure 1: Séverac et al., Phys. Fluids. 


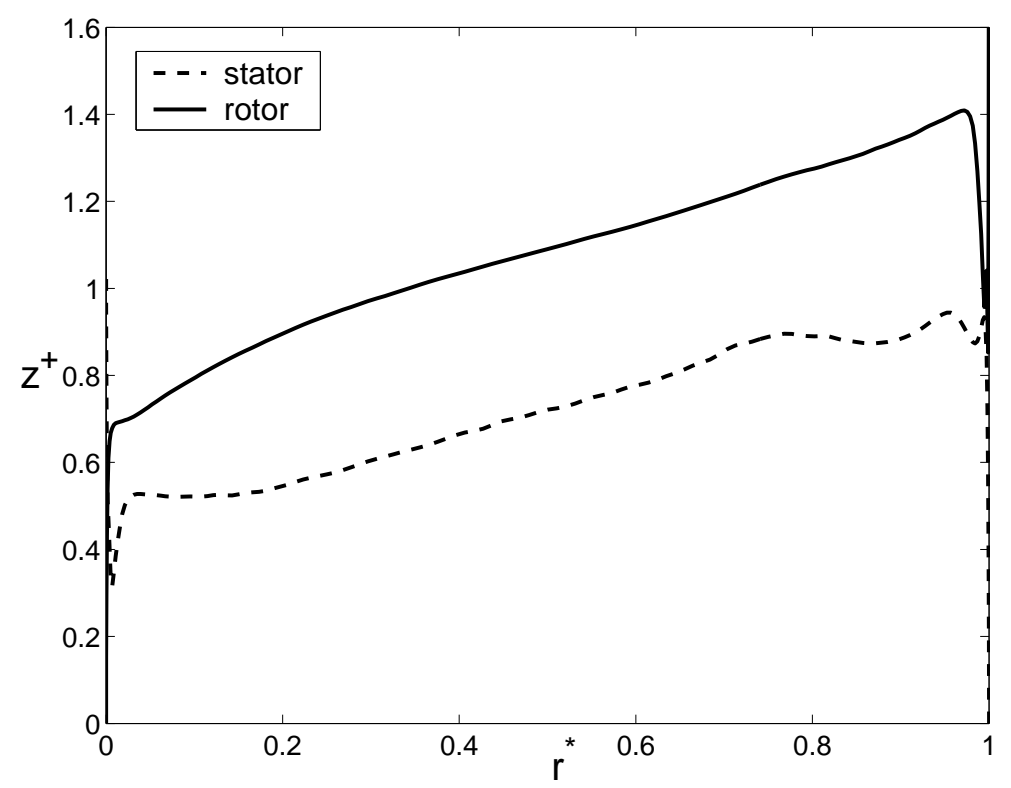

Figure 2: Séverac et al., Phys. Fluids. 

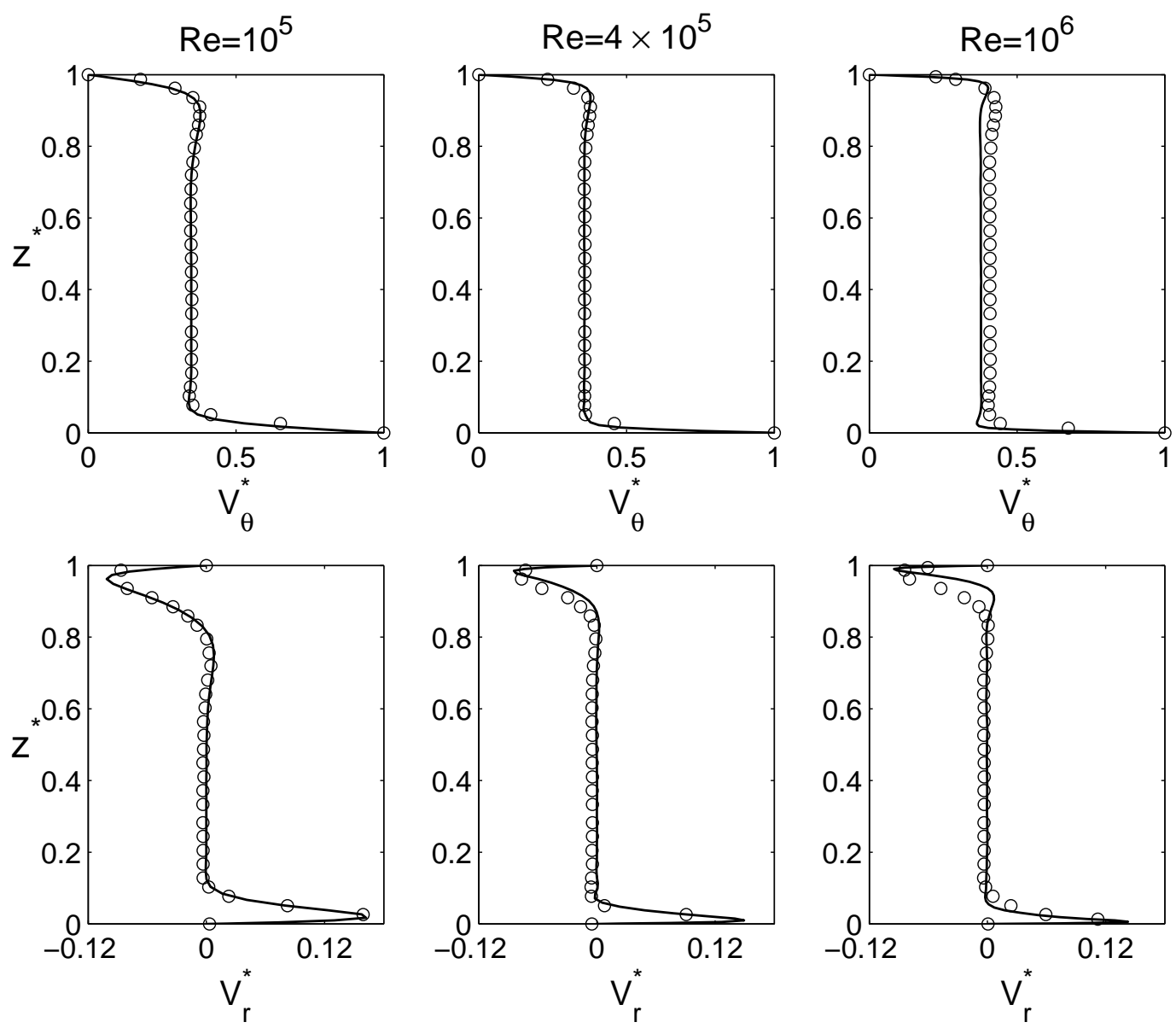

Figure 3: Séverac et al., Phys. Fluids. 

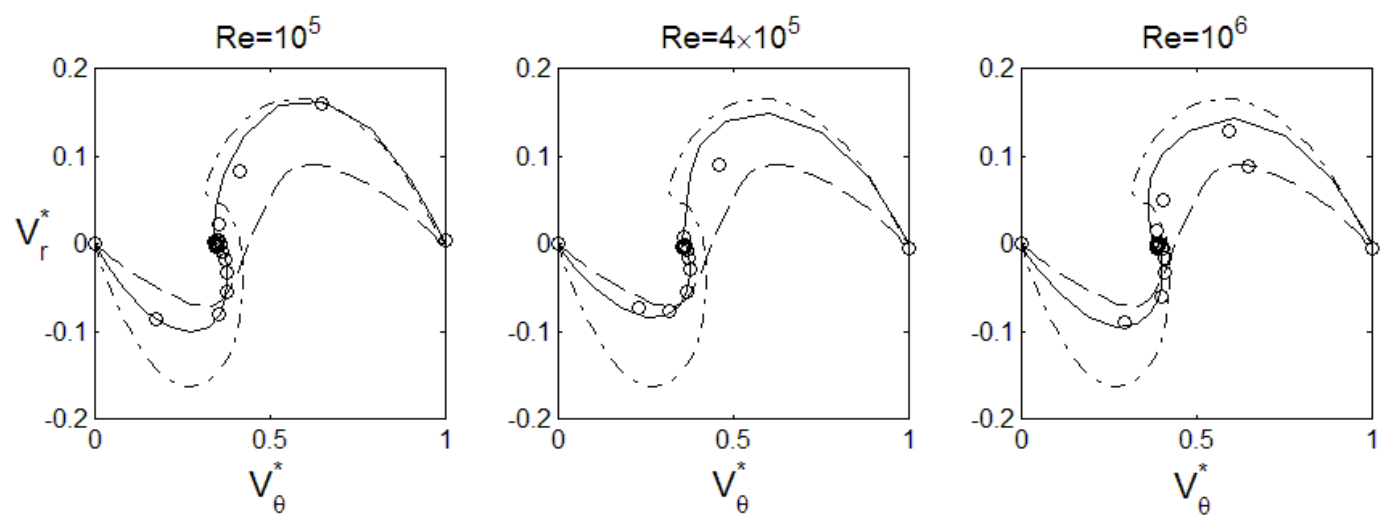

Figure 4: Séverac et al., Phys. Fluids. 


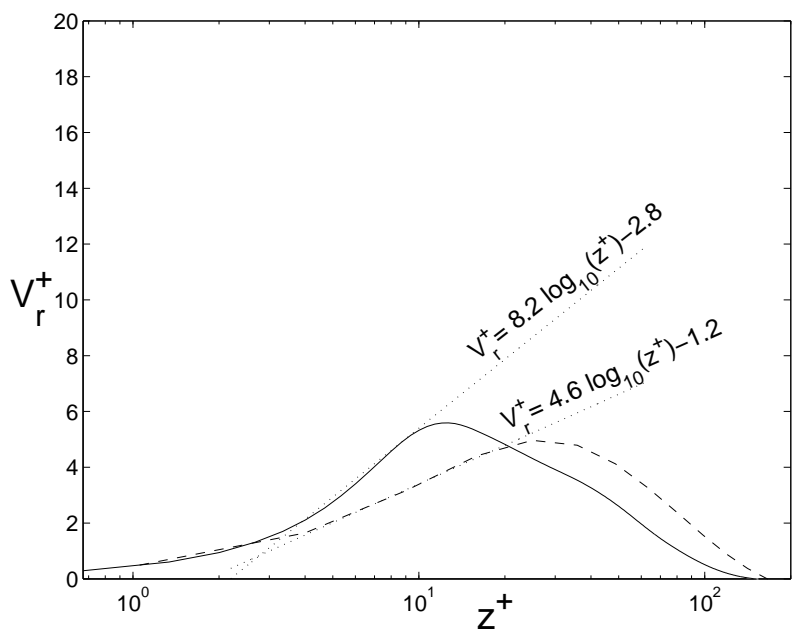

(a)

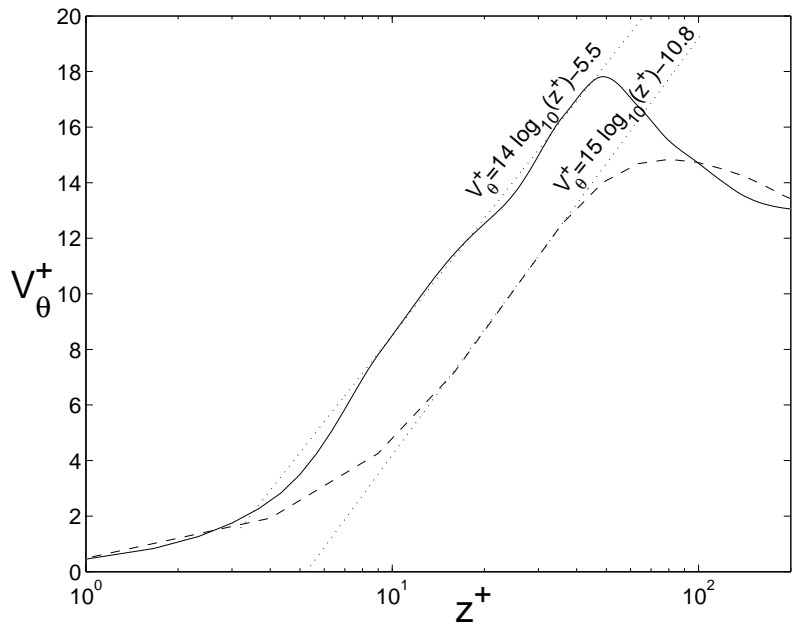

(b)

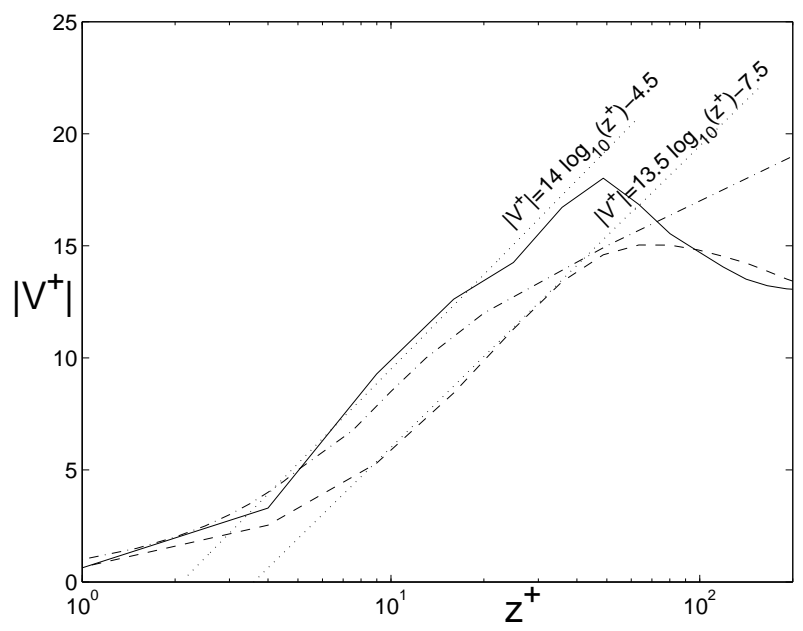

(c)

Figure 5: Séverac et al., Phys. Fluids. 

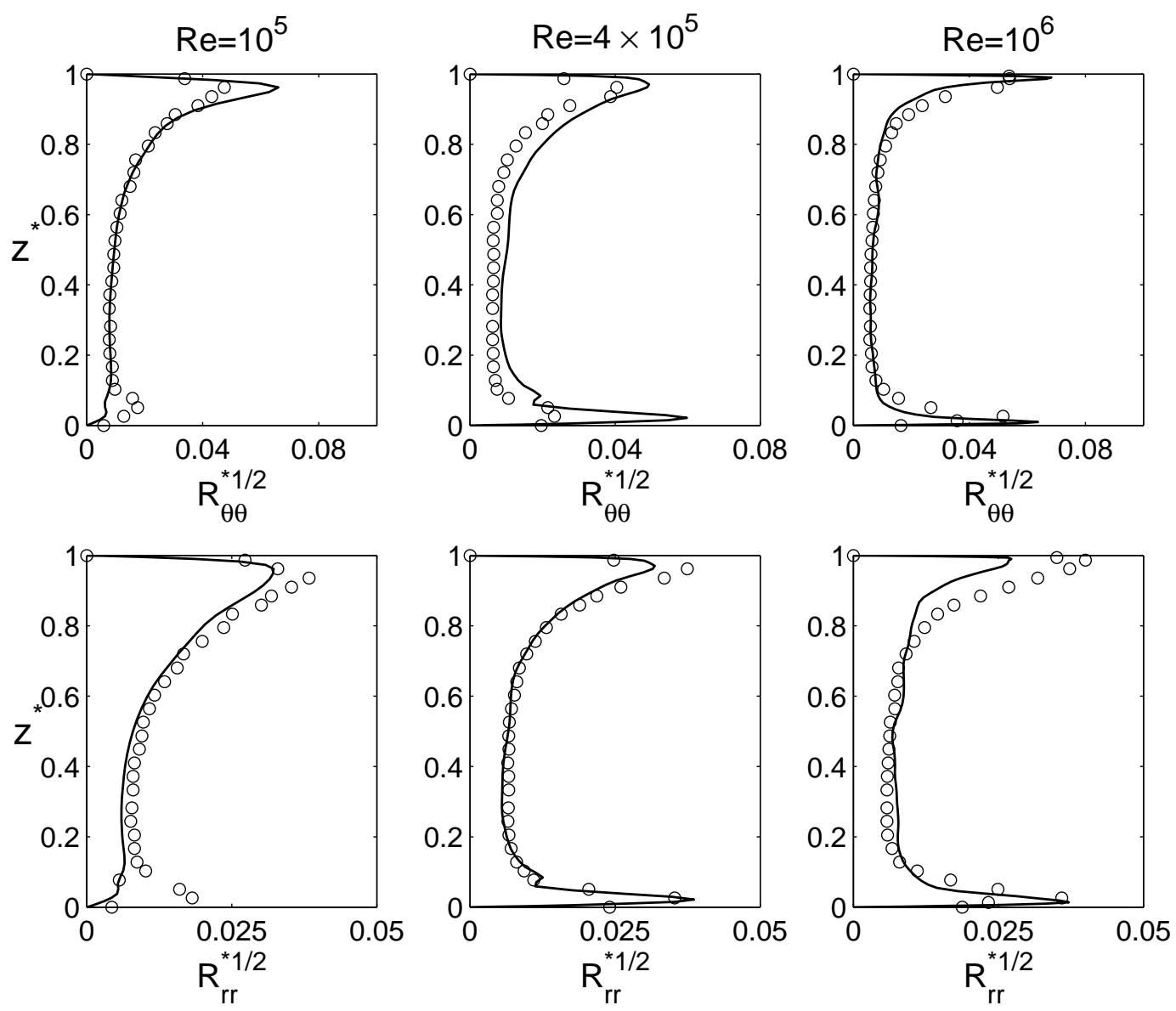

Figure 6: Séverac et al., Phys. Fluids. 

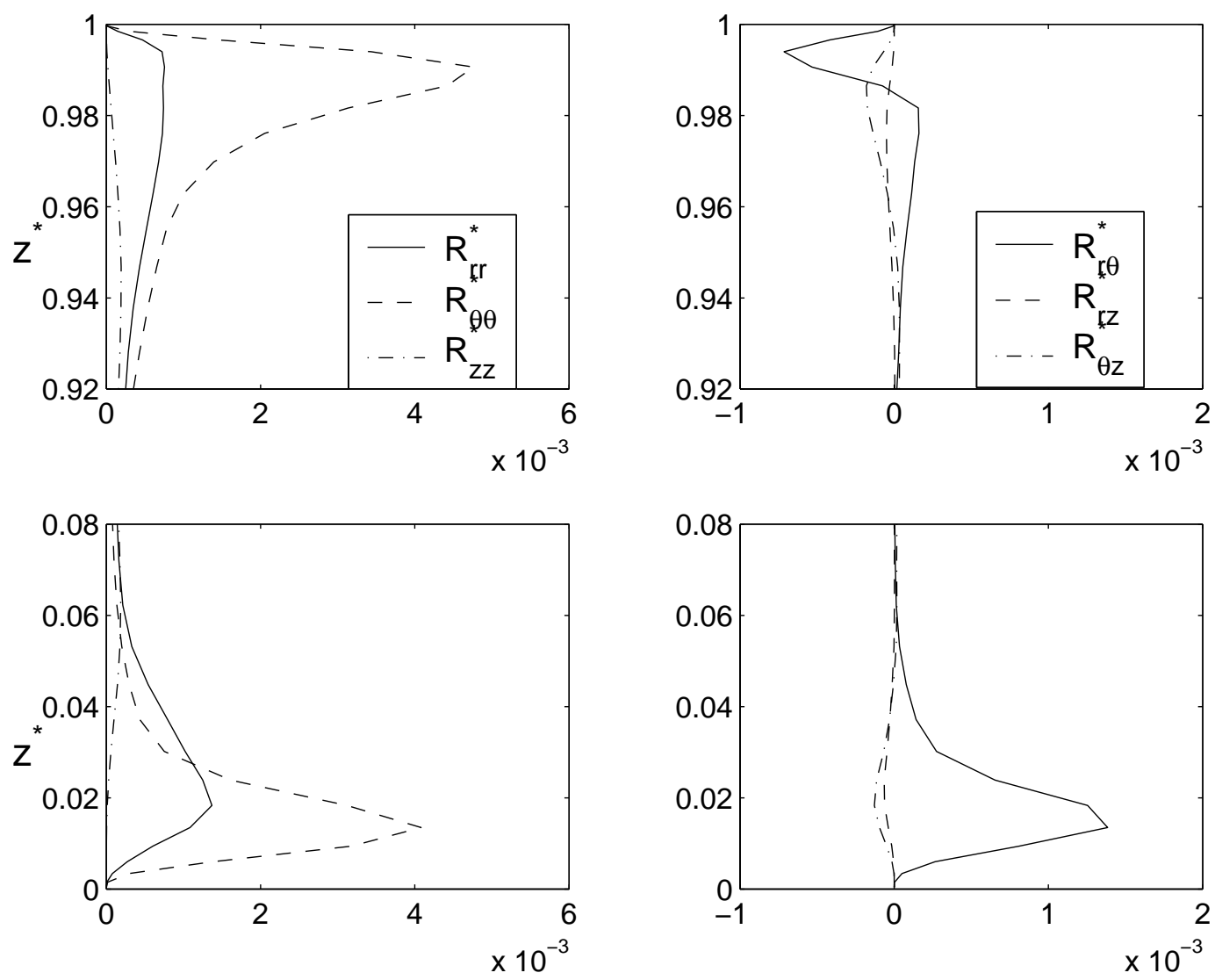

Figure 7: Séverac et al., Phys. Fluids. 


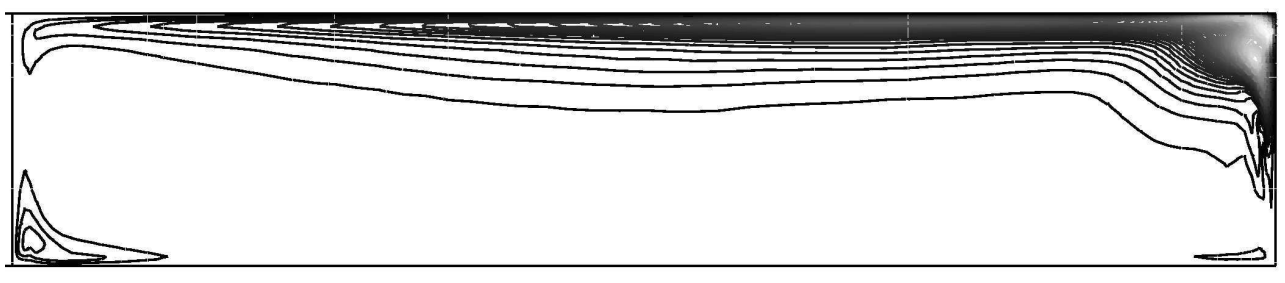

(a)
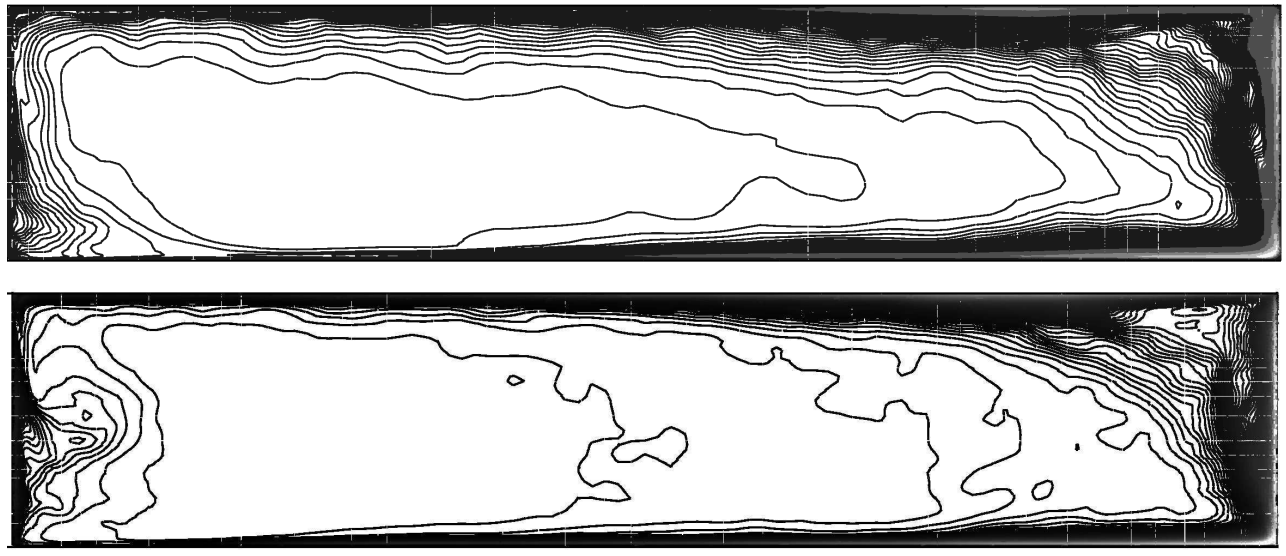

(c)

Figure 8: Séverac et al., Phys. Fluids. 

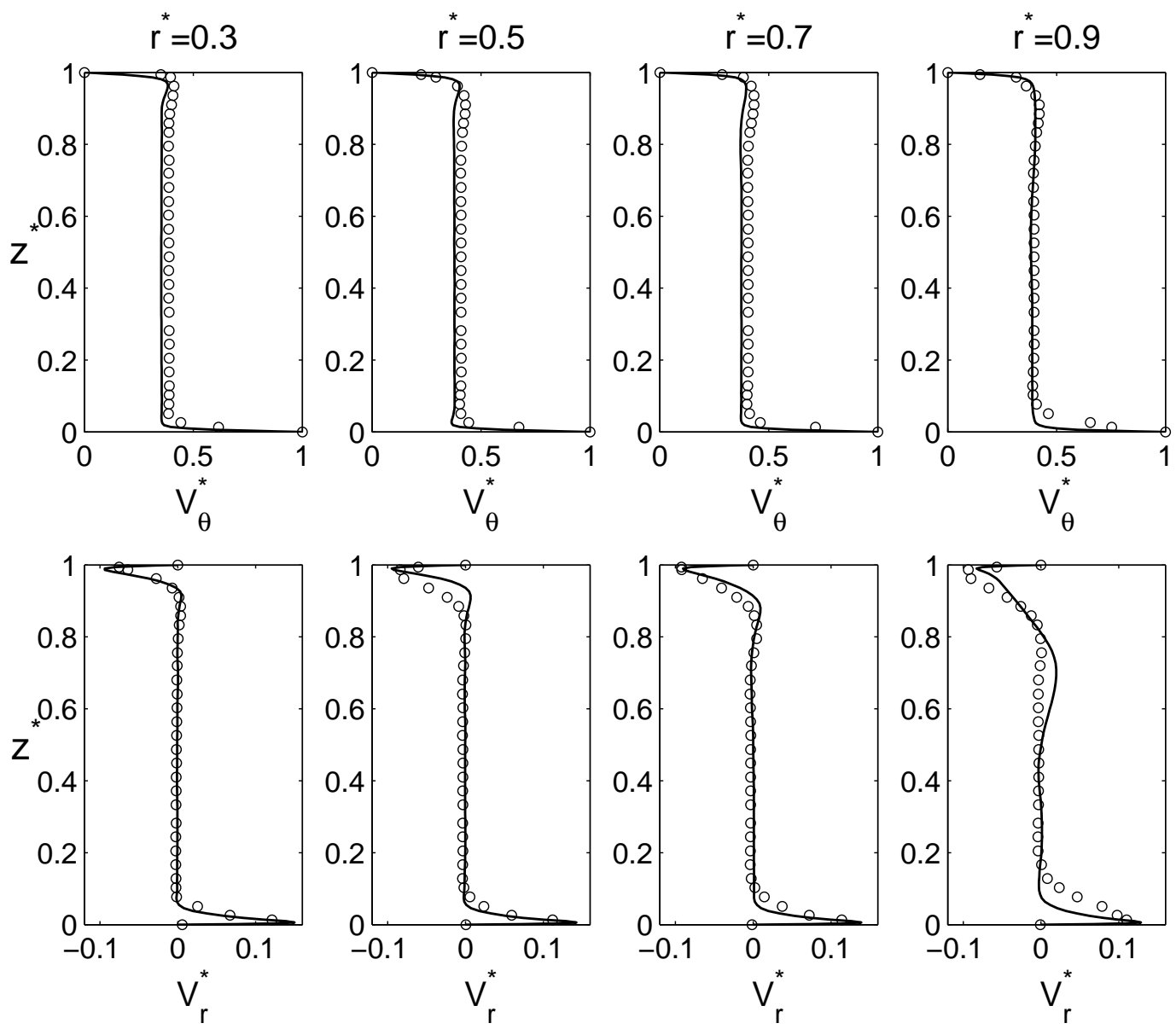

Figure 9: Séverac et al., Phys. Fluids. 


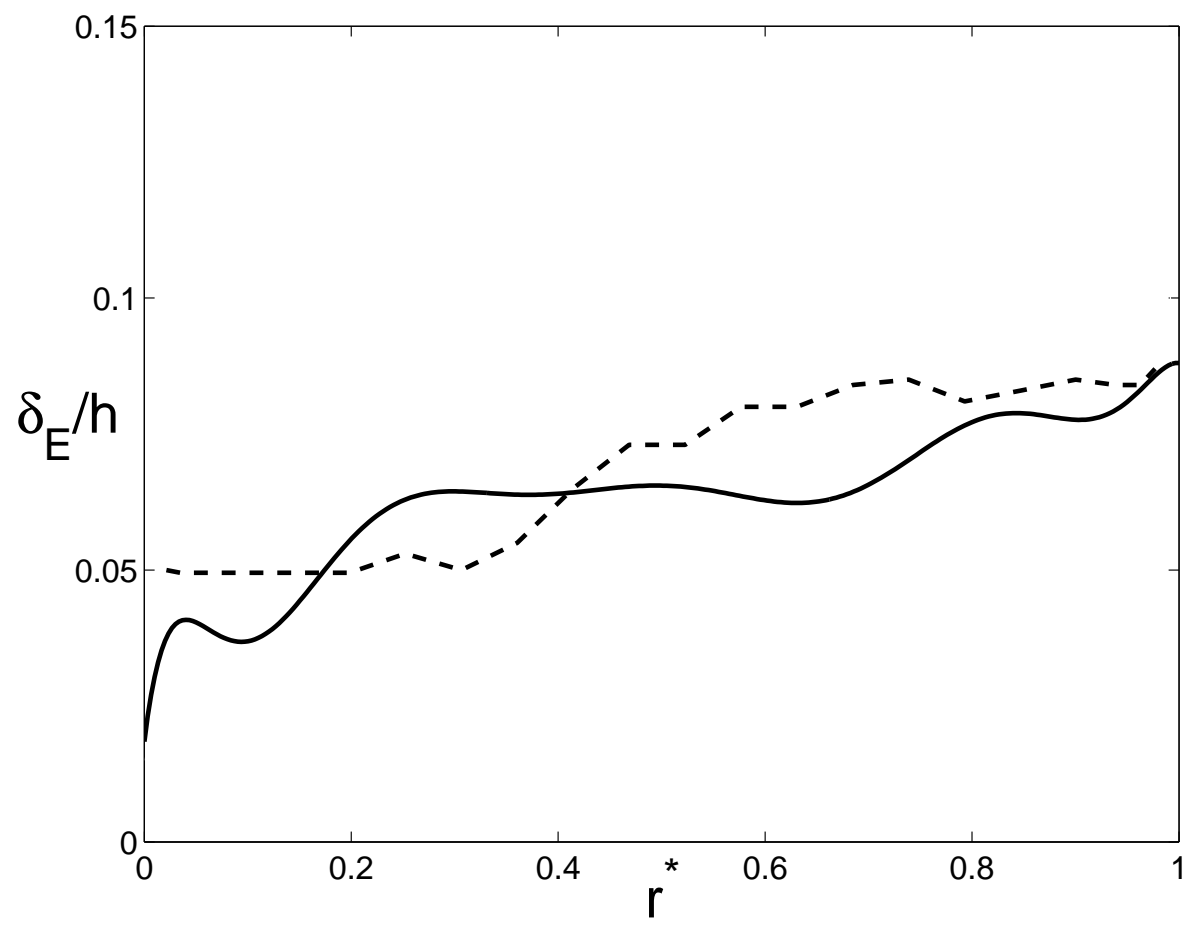

(a)

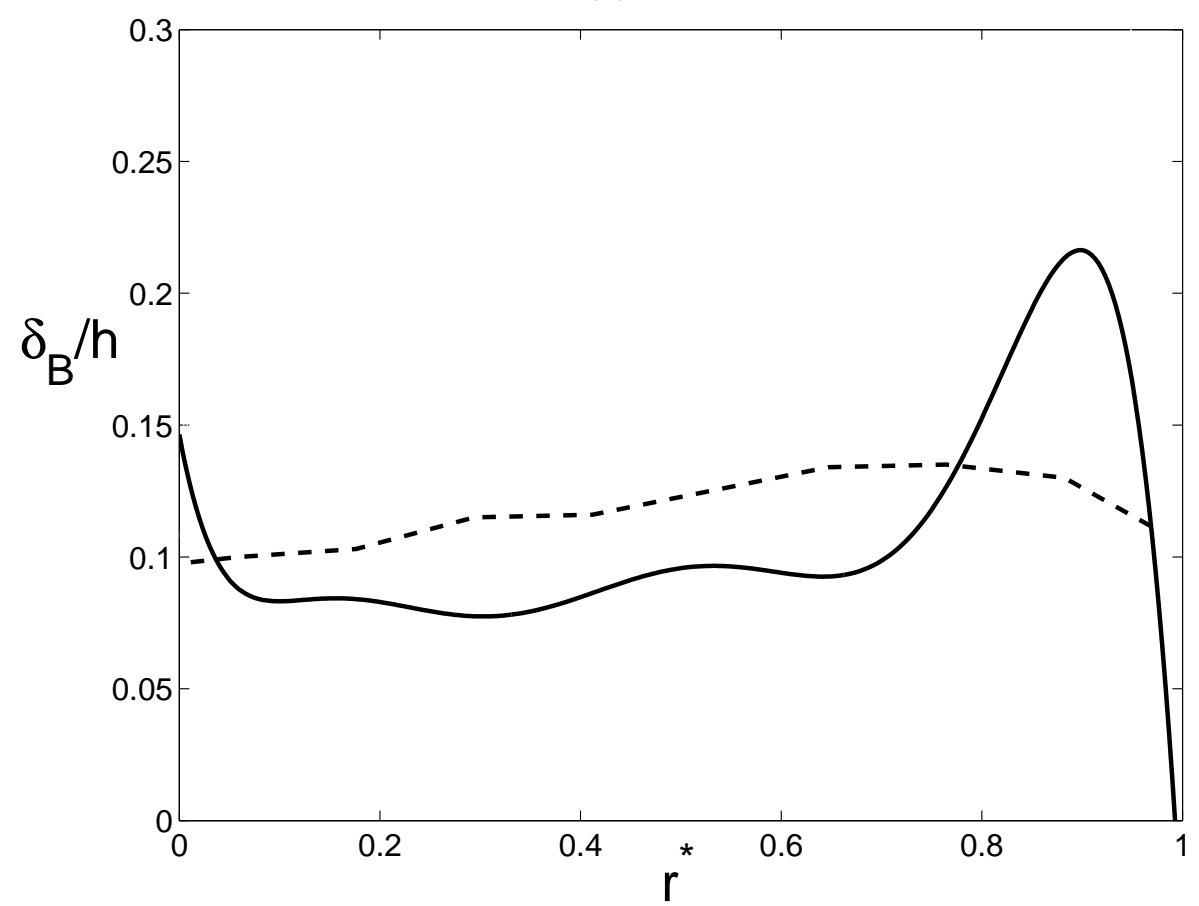

(b)

Figure 10: Séverac et al., Phys. Fluids. 


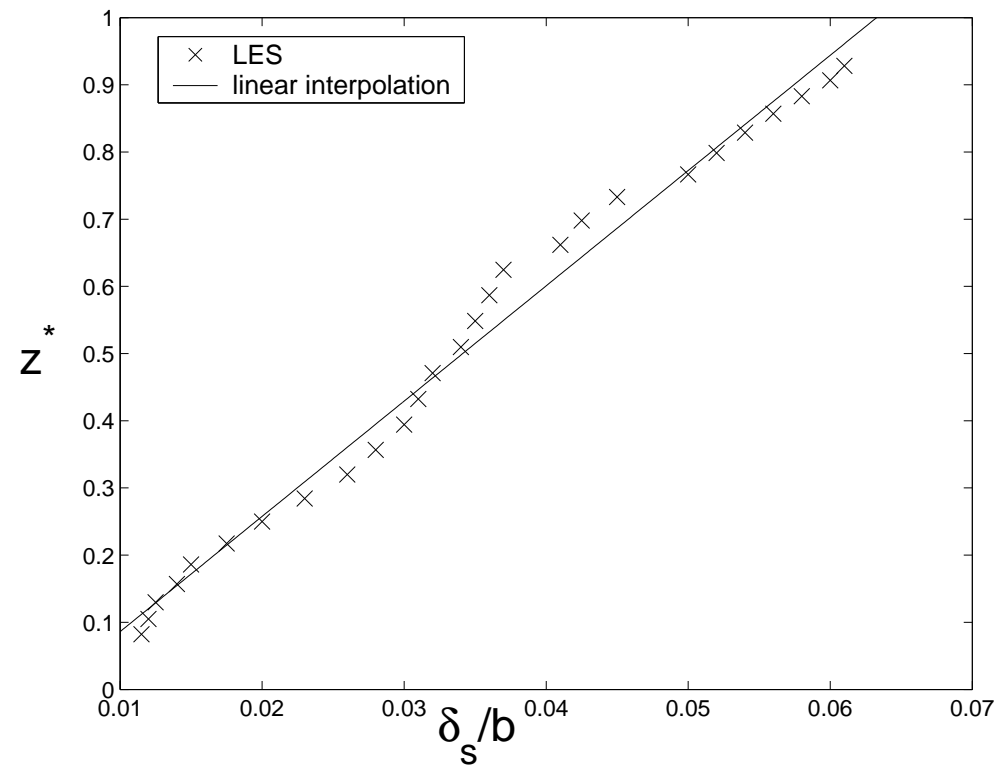

Figure 11: Séverac et al., Phys. Fluids. 

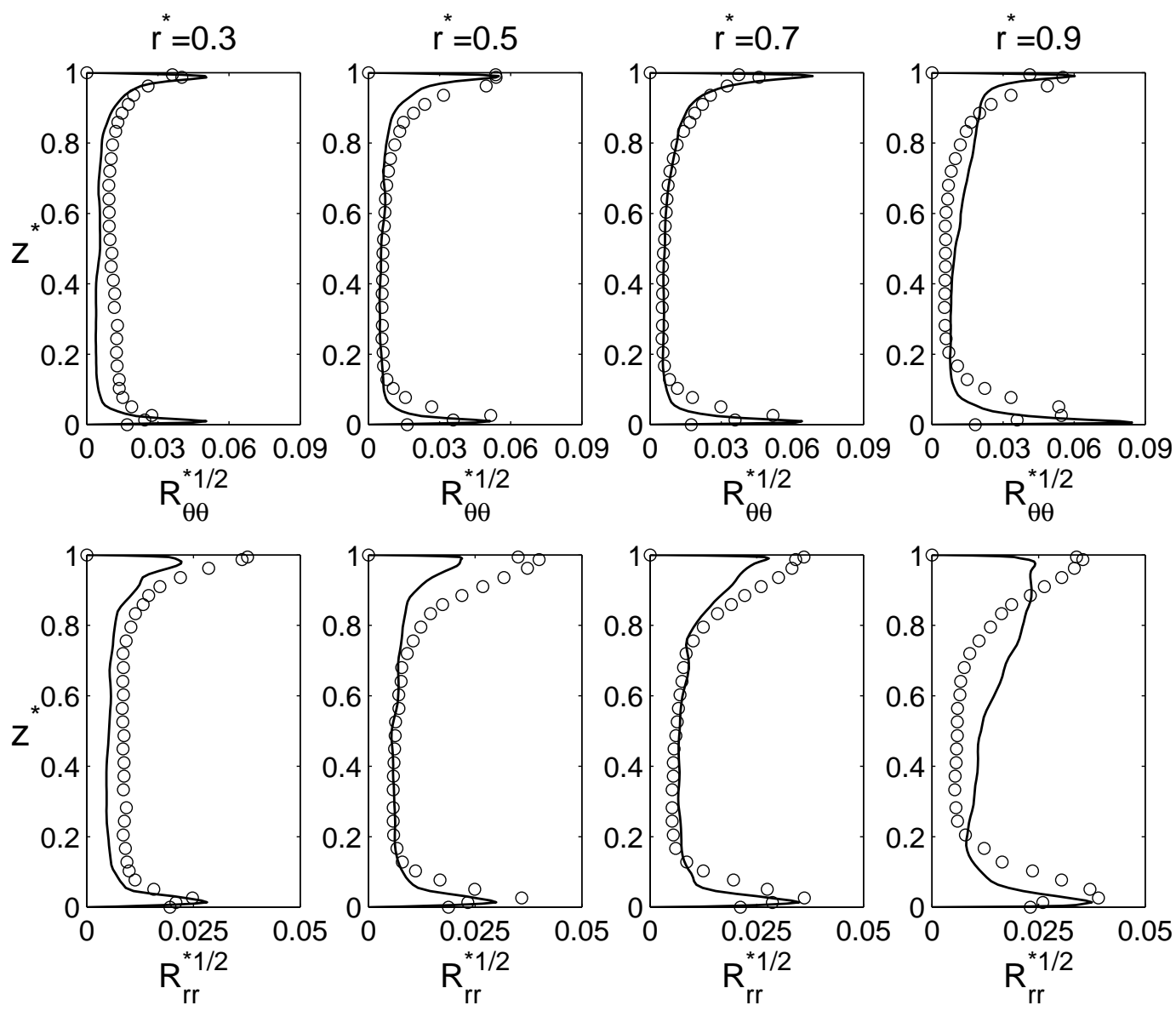

Figure 12: Séverac et al., Phys. Fluids. 

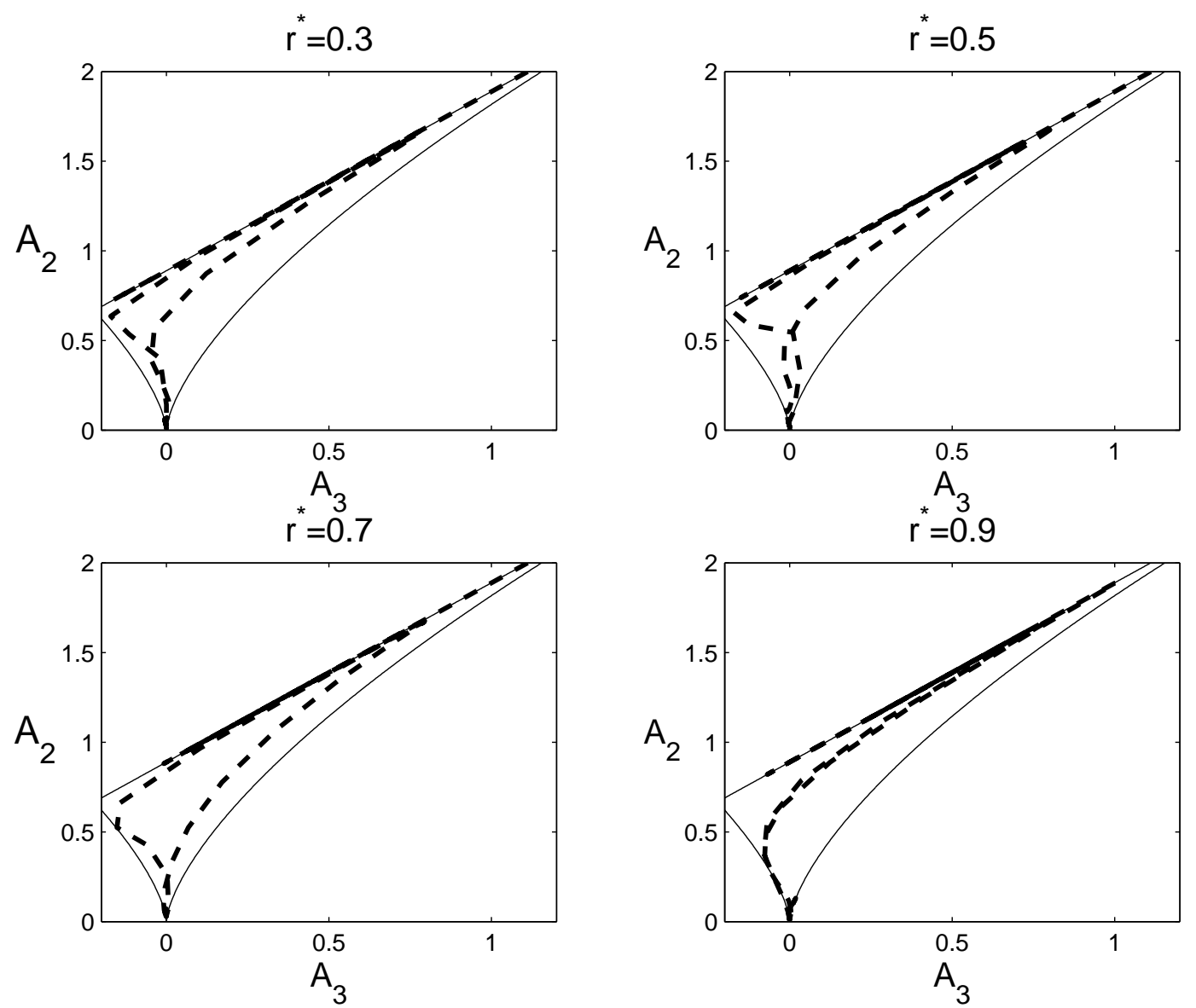

Figure 13: Séverac et al., Phys. Fluids. 


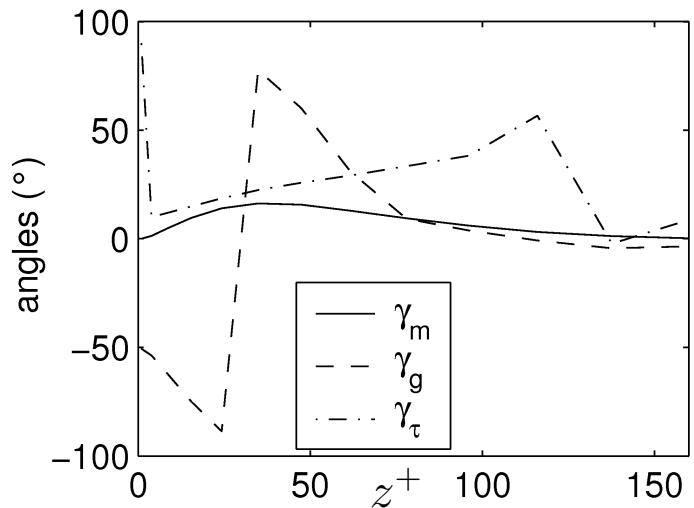

(a)

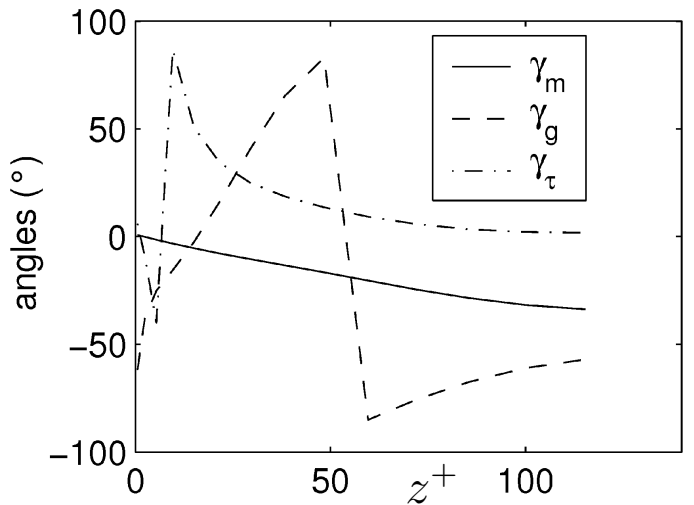

(b)

Figure 14: Séverac et al., Phys. Fluids. 

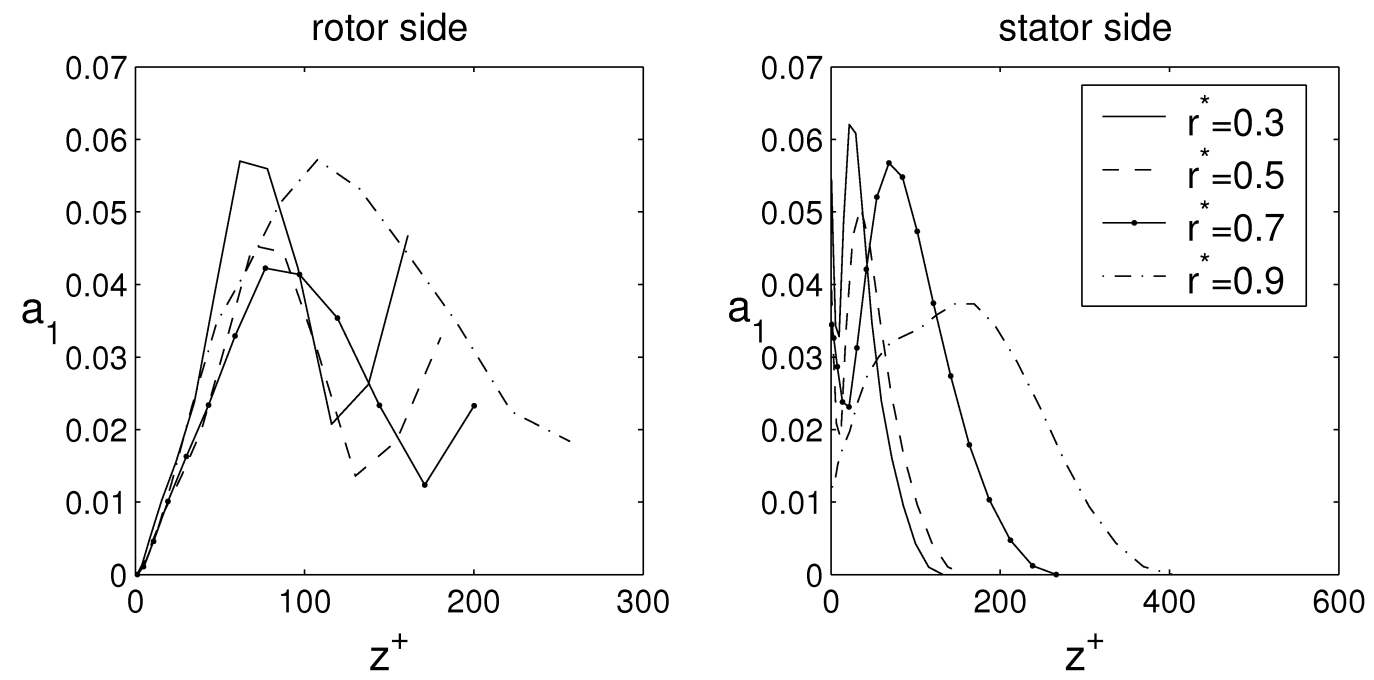

Figure 15: Séverac et al., Phys. Fluids. 


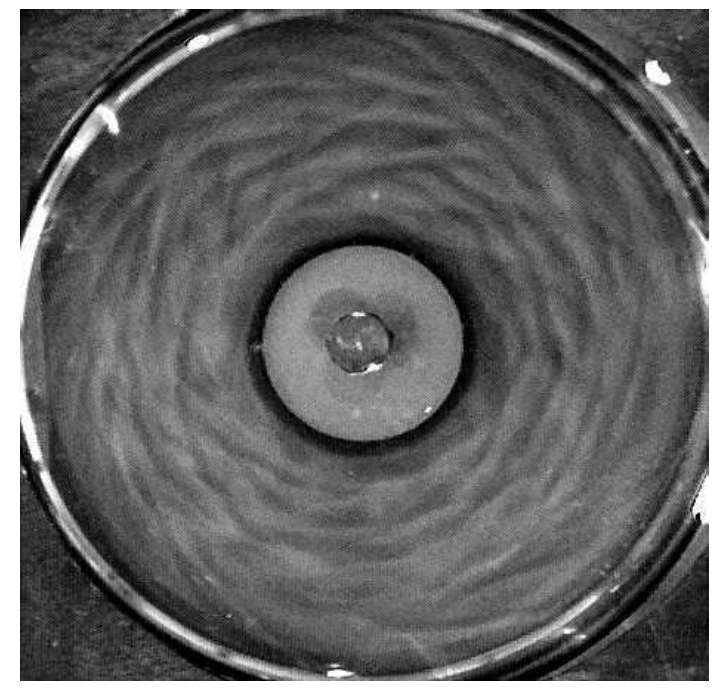

(a)

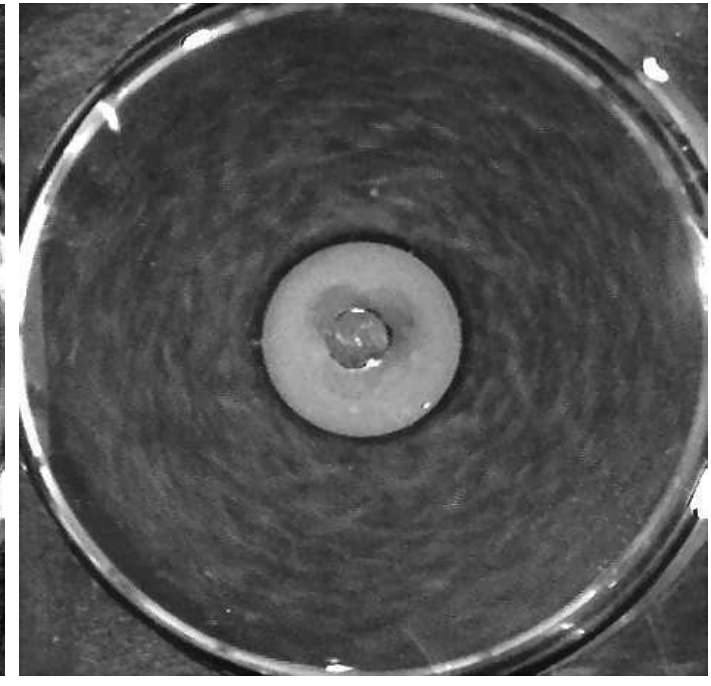

(b)

Figure 16: Séverac et al., Phys. Fluids. 
ROTOR

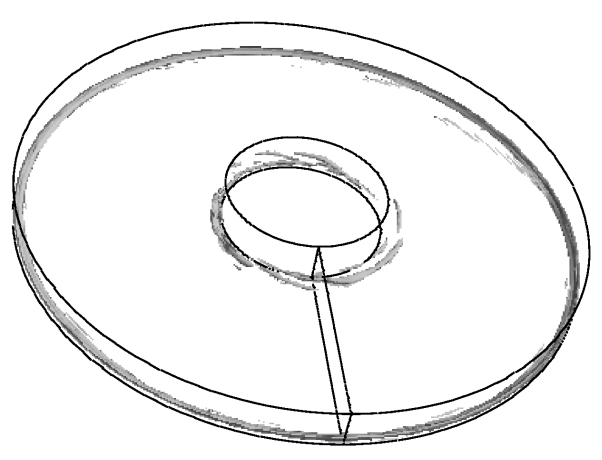

(a)

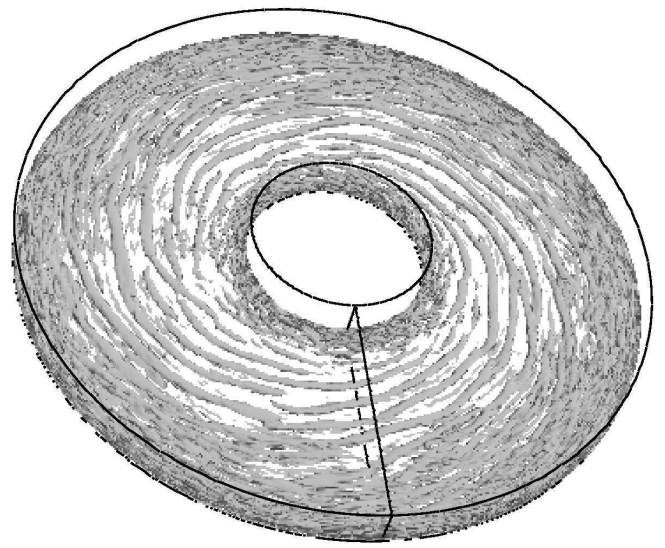

(c)

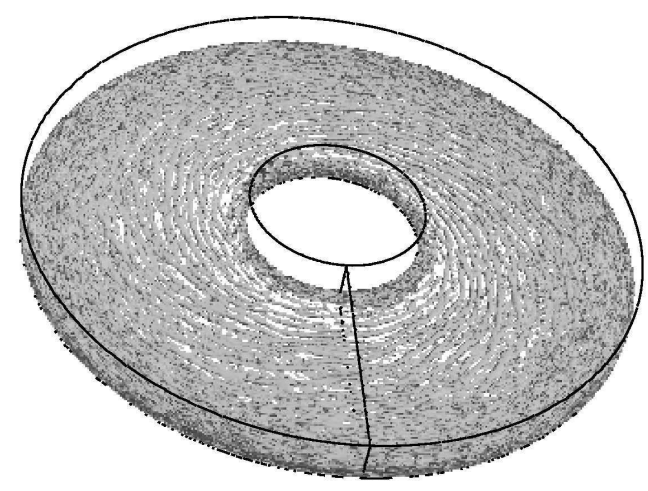

(e)
STATOR

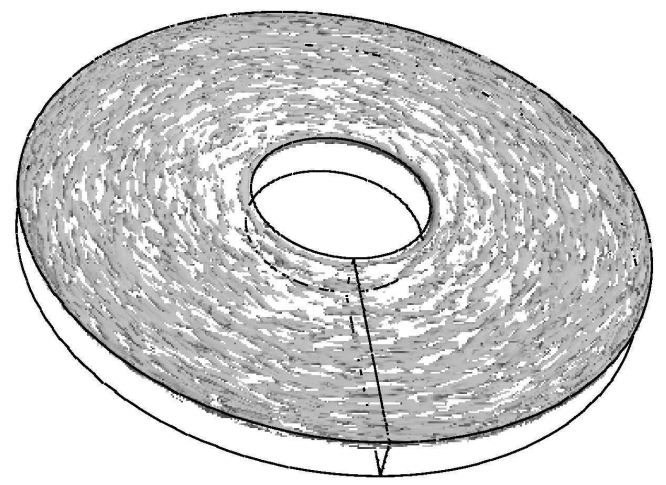

(b)

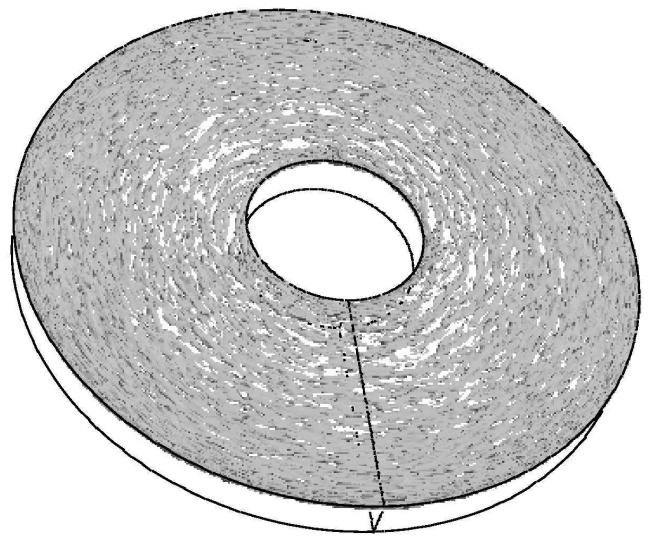

(d)

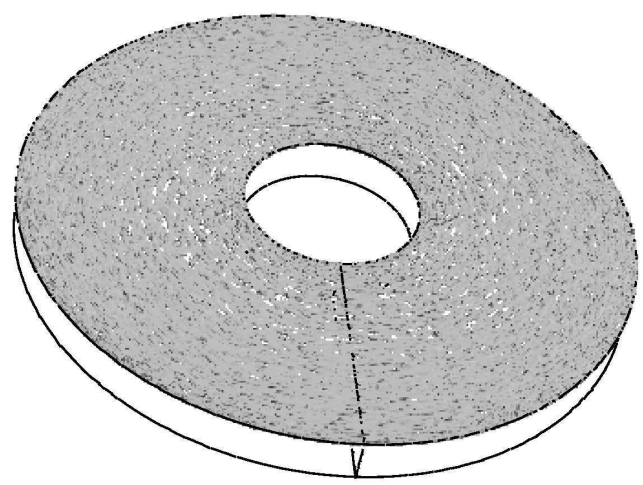

(f)

Figure 17: Séverac et al., Phys. Fluids. 